To appear in the Journal of Hydraulic Research

Vol. 00, No. 00, Month 20XX, 1-24

Research paper

\title{
Waves plus currents crossing at a right angle: near-bed velocity statistics
}

C. FARACI, Associate Professor, University of Messina, Department of Engineering, C.da di Dio, 98166

S. Agata (ME), Italy

Email: cfaraci@unime.it (author for correspondence)

P. SCANDURA, Associate Professor, University of Catania, Department of Civil Engineering and Architecture,v.le A, Doria, 6, 95125, Catania, Italy

R.E. MUSUMECI, Assistant Professor, University of Catania, Department of Civil Engineering and Architecture,v.le A, Doria, 6, 95125, Catania, Italy

E. FOTI, Full Professor, University of Catania, Department of Civil Engineering and Architecture,v.le A, Doria, 6, 95125, Catania, Italy

\begin{abstract}
Wave-current flow over a bottom covered with different roughness elements was analysed to provide new insights into the statistical properties of the near-bed velocity. Experimental data were used from three different experimental campaigns, with orthogonal waves and currents over a sandy bed, a gravel bed and a rippled bed. Velocity profiles were acquired by means of a micro-ADV. The paper focuses on the effects that the waves have on the velocity statistics of the steady current. In particular, the near-bed velocities in the current direction closely follow a Gaussian distribution. When waves are added, the distribution becomes double-peaked. In order to get single-peaked velocity distributions the total velocity events in the current direction were decoupled by taking into account the sign of the wave directed velocities. The nature of the distribution functions is influenced by the mass conservation principle and, in the rippled bed case by the vorticity dynamics.
\end{abstract}

Keywords: Bed roughness; higher-order statistics; probability distribution function; ripples; wave boundary layer; wave-current interaction.

\section{Introduction}

In shallow coastal waters the near bottom flow often consists of waves and slowly varying currents, the latter being generated by phenomena such as radiation stress, set-up, tides or other factors. More specifically, it has been observed that the thin wave boundary layer at the bottom, driven by the oscillating wave orbital velocity, strongly affects coexisting currents (Grant \& Madsen, 1979). Indeed the high shear velocity within the wave bottom boundary layer generates strong turbulence and large bed shear stresses which impact on the current, leading to increased bottom resistance in the presence of combined flows (Lodahl, Fredsøe, \& Sumer, 1998). The combined wave-current flow thus plays a fundamental role on the sediment transport, mixing processes, diffusion, and other important coastal phenomena.

Several researchers contributed to the understanding of the physical mechanisms of wave-current interaction, by analysing the combined flow over smooth or rough beds both experimentally or numerically. Most of these studies are focused on the effects of the mutual interaction of the two flow components on the velocity profile, particularly within the bottom boundary layer.

The effects of waves following or opposing the current over fixed smooth or rough beds have been widely investigated. For example, Simons, Grass, and Mansour-Tehrani (1992) and Simons, 
Grass, Saleh, and Mansour-Tehrani (1994) analysed the case of waves propagating with or against a current over a fixed layer of sand, and found that the presence of waves induces a significant reduction of the mean flow in the upper part of the water column and an increase close to the bed. Lodahl et al. (1998) observed that when an oscillatory flow colinear with a current is current dominated, a linear interaction between the laminar wave and the current occurs; if the flow is wave dominated, a relaminarization of the steady turbulent flow or an increase of the shear stress may occur, depending on whether the boundary layer is laminar or turbulent. Huang and Mei (2003) developed a theoretical model to predict the wave influence on a turbulent current over smooth or rough beds. They determined a velocity increase in the case of waves propagating with the current and a decrease in the case of opposing flows. Recently, Yuan and Madsen (2015) measured the horizontally uniform turbulent wave-current bottom boundary layer flow subject to asymmetric forcing. They found that within the seabed boundary layer flow the magnitude of the mean velocity is larger for opposing than for following waves and current.

In the field, particularly in the nearshore region, waves and currents interact at an angle, often close to $90^{\circ}$. The experimental and numerical investigation of the interaction of waves and currents superimposed at an angle is much more difficult and far fewer studies exist. Arnskov, Fredsøe, and Sumer (1993) performed experiments on waves and currents interacting at $90^{\circ}$ over a smooth bed to evaluate the effects of the flow interaction on the bed shear stress. In this case significant nonlinear enhancement of the maximum shear stress was not observed. Andersen and Faraci (2003) used the same experimental setup with a movable bed to detect the range of wave - current velocities where bedform geometry could be assumed to be forced only by the oscillatory flow, in order to validate some assumptions in a $\kappa-\omega$ turbulence closure model.

Musumeci, Cavallaro, Foti, Scandura, and Blondeaux (2006) investigated the flow produced by waves plus current crossing at a right angle over rough beds. They found that when waves are added onto a current over a bed characterised by a small roughness, an increase of the current flow at the bed occurs; the contrary occurs in the case of large roughness. Orthogonal waves and current over a movable bed were also considered by Fernando, Guo, and Lin (2011), who collected velocity measurements and compared their results with existing literature models for wave-current interaction. They found that an agreement between models and experiments exists only for small to medium wave heights. Lim, Madsen, and Cheong (2012) in combined flows documented an increase of the angle between the mean flow and the waves due to the presence of wave induced mass transport and a decrease of the hydraulic roughness with respect to the current alone case. Similar results have been obtained by Lim and Madsen (2016).

Waves plus currents over rippled beds have been investigated, among others, by Ranasoma and Sleath (1994), Mathiesen and Madsen (1996), Fredsøe, Andersen, and Sumer (1999), Faraci, Foti, and Musumeci (2008) and Madsen, Negara, Lim, and Cheong (2010). Ranasoma and Sleath (1994) performed LDA measurements of orthogonal wave-current flow over fixed ripples. Close to the bed they found that due to the momentum exchange induced by the vortex ejection, measurements do not agree with classical eddy viscosity models. Mathiesen and Madsen (1996) compared the wave roughness with that of a combined flow, and found that in the case of combined flow the roughness is similar to that of a pure wave and a single roughness scale can be considered. Fredsøe et al. (1999), by means of both experimental results and a $\kappa-\omega$ turbulence closure model, found an increase up to one order of magnitude in the bed roughness when waves are superimposed to a current, while Faraci et al. (2008) found that a rippled bed behaves like a macro-roughness towards the current, causing the wave boundary layer to become turbulent. Madsen et al. (2010) observed that when wave induced bedforms are at an angle with the current, the near bottom flow tends to veer parallel to the ripple axis; a dramatic increase of the roughness experienced by a rippleparallel current is also observed when near perpendicular waves are present, due to the non-linear interaction of the velocity components.

Notwithstanding the large amount of literature on wave-current interaction, the attention has been focused almost exclusively on the mean flow, whereas to the authors' knowledge little has been 
reported to understand the statistics of the combined flow, which, due to turbulence, fluctuates spatially and temporally around its average values. Nevertheless, a quantitative analysis of the statistical properties of a combined wave-current flow is necessary to understand some aspects of important bed processes, such as erosion and sediment transport. For example, it is known that the threshold of sediment motion is mainly due to the peak velocities of the turbulent flow which destabilizes the bed particles, but it is often referred to the mean shear stress at the bottom. A pioneering work in this context was performed by Grass and Ayoub (1983), who proposed a probability based model to interpret turbulence induced instability of a movable bed. Later on Parker, Paola, and Leclair (2000) also reported that the implementation of a probabilistic concept in reformulating Exner equation provides a better understanding of the sediment transport at the bed. The importance of using a probabilistic approach to describe the bed characteristics themselves has been also recognized. For example, Nikora, Goring, and Biggs (1998) and Friedrich, Nikora, Melville, and Coleman (2006) used a random field approach to characterize a gravel or a rippled bed as an alternative to the characteristic particle size approach, and found that the gravel bed elevation is distributed as a Gaussian-like variable, while the coupling of skewness and flatness values allows different kinds of bedforms to be distinguished.

Although the characterization of the flow is desirable, as pointed out by Cheng (2006), the majority of the works dealing with sediment transport problems takes into account the turbulence only through a time averaged velocity or shear stress. Among the few studies which have shed light on the properties of turbulence, Kim, Moin, and Moser (1987) performed a DNS of a turbulent channel flow in the presence of smooth walls, to obtain quantitative information on turbulence structure. Recently, time resolved PIV techniques have been employed by Willert (2015) for estimating flow statistics, spectra, probability density functions and correlations of a turbulent boundary layer in a wind tunnel. However none of the previously mentioned works deal with combined wave-current flows.

In order to contribute to the characterization of such type of flows, the present work reports on an analysis of both low-order and high-order statistics of the velocity field generated by superimposing a regular wave field to an orthogonal current. In order to understand the role played by the bed roughness, three different fixed bottom conditions are considered, namely a sandy bed, a gravel bed and a rippled bed. The present analysis is performed using an existing experimental dataset (Faraci et al., 2008; Musumeci et al., 2006), acquired by the authors but never analysed in this perspective. In particular, this study is focused on the influence of the waves on the current properties. The distribution functions of the velocity in the current direction, considering both the total velocity and the turbulent fluctuations, are discussed. Moreover, since it is recognized that the wave phase plays a major role on the modification of the current, the effects of the combined flow on the phase-averaged flow are also presented.

The paper is organized as follows: first the experimental set up and procedure are presented, then experimental data are discussed in section 3 and the conclusions are presented in section 4 .

\section{Experimental campaign}

\subsection{Experimental set up and procedure}

The experiments were carried out at the Hydraulic Laboratory of the University of Catania in a wave flume equipped with a recirculating system which allows a uniform current to enter the flume at a right angle with respect to the direction of wave propagation.

The wave flume is $18.00 \mathrm{~m}$ long, $4.00 \mathrm{~m}$ wide and $1.20 \mathrm{~m}$ deep. Regular and irregular waves can be generated by a flap type wavemaker, which is driven by an electronically controlled pneumatic system. Opposite the wavemaker a porous plane beach limits the reflection to about $10 \%$ of the incident wave. For a detailed description please refer to Faraci et al. (2008) or Faraci, Foti, Marini, 
and Scandura (2012).

The current is driven by a submerged $11.0 \mathrm{~kW}$ electropump, with a maximum discharge of 0.25 $\mathrm{m}^{3} / \mathrm{s}$; it flows within a series of channels, in order to dampen turbulence, and it enters the wave flume at a right angle. The current inlet is $2.5 \mathrm{~m}$ wide, the outlet is perfectly mirrored with respect to the inlet. The corners of the inlet and of the outlet are shaped in such a way as to minimize effects of wave diffraction on the current within the measurement area. Additionally, passive wave absorbers at the outlet walls minimize spurious wave reflections.

Three different types of bed roughness have been considered in the central part of the wave flume, covering an area of $3.5 \mathrm{~m} \times 4 \mathrm{~m}$. During the first campaign, referred to as SB, a single layer of fine quartz sand characterized by a $D_{50}$ of $0.24 \mathrm{~mm}$ was glued onto the bed; during the second campaign, referred to as GB, a single layer of marble stones with a median grain size of $D_{50}=30$ $\mathrm{mm}$ was glued on the bed to simulate a gravel bed. These two campaigns have been extensively described in Musumeci et al. (2006). Finally the third campaign, referred to as RB, was carried out by covering the bed with a fixed $2 \mathrm{D}$ PVC rippled panel, made up by a unique contoured block. The regular ripple height was $1.85 \mathrm{~cm}$ and ripple length was $12.5 \mathrm{~cm}$. The bedform dimensions were chosen in such a way as to be in agreement with the characteristics of ripples generated during the experimental campaign performed by Andersen and Faraci (2003), in the presence of orthogonally superimposed waves and currents characterized by the same ratio between current velocity and wave orbital velocity. In particular it was found that the chosen bed form characteristics are typical for waves with periods between 1 and $1.4 \mathrm{~s}$ and heights of about $0.1 \mathrm{~m}$ propagating over a depth of about $0.3 \mathrm{~m}$. The detailed experimental data of the RB campaign have been reported in Faraci et al. (2008).

For each type of bed roughness, three different types of flows have been generated: wave-only, current-only and waves plus currents. The surface elevation was measured by means of several resistance wave gauges located along the flume. Moreover, a Sontek micro Acoustic Doppler Velocimeter (micro-ADV), mounted on a movable carriage, was used to measure the three velocity components. The sampling volume is a cylinder with a height of $9 \mathrm{~mm}$ and a volume of $0.3 \mathrm{~cm}^{3}$, located $5 \mathrm{~cm}$ below the transmitter. Due to the dimensions of the measuring volume, the closest point to the bed was located about $0.45 \mathrm{~cm}$ above the bottom. Thus velocity measurements were not gathered either within the thin wave bottom boundary layer $(\mathrm{O}(\sim 1 \mathrm{~mm}))$, and in the region between wave crest and $5 \mathrm{~cm}$ below the wave trough. The adopted sampling frequency was $30 \mathrm{~Hz}$.

In Musumeci et al. (2006) a detailed report on the spatial homogeneity of the wave current interaction in the central part of the channel is reported. Within this area a single measurement station located along the mid line of the current stream, $2.5 \mathrm{~m}$ far away from the inlet, has been considered for the SB and GB campaigns. For RB campaign five measuring stations were considered along the ripple profile, two of them at the two adjacent crests, one at the trough and the other two between crest and trough (see Fig. 1b). Each velocity time series was acquired for $\approx 60$ wave cycles. In order to verify that the sampling time was long enough, it was ascertained that the standard deviation of the current velocity component was stable. In Table 1 this quantity is reported for the point closest to the bed in the gravel bed roughness case and for a wave plus current condition. Here it is shown that even though the acquisition has been performed for a longer time period (75 cycles), convergence can be considered to be reached after 60 cycles. Each velocity profile was obtained collecting about 10 - 15 points for each station along the water column.

Figure 1 reports a sketch of the wave plus current experimental flume where the two orthogonal interacting flows are highlighted. Figure 1 shows also the reference system, where $x$ is in the steady current direction, $y$ is in the direction of the wave propagation, $z$ is positive upward and the origin is located at the bottom in the flat bed experiments (SB and GB) and at the trough level in the rippled bed $(\mathrm{RB})$ tests.

The experimental procedure can be summarized as follows:

(1) a regular wave motion is generated until steady conditions are reached; 
Table 1 Convergence test. SB, $V_{D A}=0.067 \mathrm{~m} / \mathrm{s} ; H=0.085 \mathrm{~m} ; T=1 \mathrm{~s}$.

\begin{tabular}{cccc}
\hline no of cycles & $V_{x} \begin{array}{c}\text { standard deviation } \\
(\mathrm{cm} / \mathrm{s})\end{array}$ & $V_{x}$ skewness & $V_{x}$ flatness \\
\hline 40 & 0.7533 & 0.0331 & 2.8443 \\
60 & 0.7894 & 0.0875 & 2.9123 \\
75 & 0.7890 & 0.0858 & 2.9473 \\
\hline
\end{tabular}
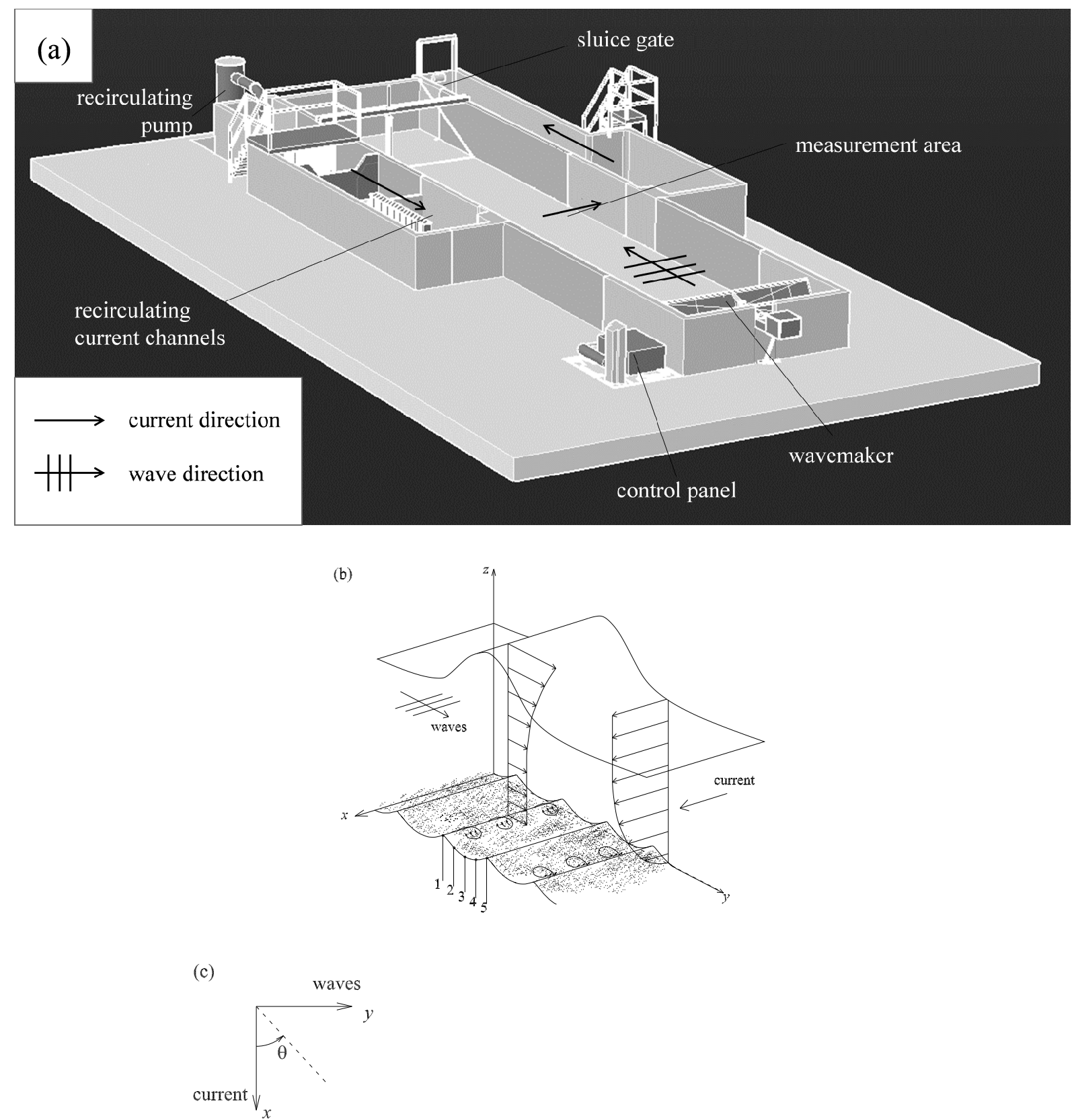

Figure 1 (a)3D sketch of the wave plus current flume used for the experimental campaigns; (b) Sketch of the used reference system, along with the vortex flow close to the rippled bed (for RB tests); (c) angle reference system. 
(2) wave characteristics and velocity profiles are gathered at the measuring stations;

(3) after stopping the wavemaker and fixing the discharge, the recirculating system is run until the water levels in all the channels are stable and a uniform current is established within the wave tank;

(4) current only velocity profiles are acquired;

(5) waves are superimposed to the existing current and wave current velocity profiles are acquired after steady conditions are reached.

Steadiness of the flow was checked by monitoring mean levels in all the channels; in the presence of currents it was usually reached after 2-3 hours after starting the apparatus.

\subsection{Experiments}

Three sets of experiments, previously denoted as SB, GB and RB campaigns, are discussed here. Wave conditions in all the experiments are characterized by periods between $0.8 \mathrm{~s}$ and $1.6 \mathrm{~s}$ and two different wave heights, namely $0.085 \mathrm{~m}$ and $0.105 \mathrm{~m}$. Water depth $D$ is kept constant in all the tests and equal to $0.3 \mathrm{~m}$, while three different values of depth averaged current velocities $V_{D A}$ are considered, namely $0.045 \mathrm{~m} / \mathrm{s}, 0.067 \mathrm{~m} / \mathrm{s}$ and $0.100 \mathrm{~m} / \mathrm{s}$. Current only, wave only and wave plus current flow conditions have been considered.

Table 2 reports a summary of the experimental conditions, where the $\mathrm{X}$ mark in the bottom roughness columns denotes a performed test. For each test the vertical profiles of the three velocity components, $V_{x}, V_{y}$ and $V_{z}$ in the $x, y$ and $z$ directions have been acquired.

\section{Analysis of experimental data}

The analysis of the experimental data is carried out here in order to provide information on the statistics of the velocity component $V_{x}$ in the direction of the current. In general all the velocity components can be presented as the sum of two terms:

$$
V_{x}=<V_{x}>+v_{x}^{\prime}
$$

where $\left\langle V_{x}\right\rangle$ is the ensemble averaged velocity, and $v_{x}^{\prime}$ is the turbulent fluctuating velocity. The ensemble average can be further decomposed into two terms as shown below:

$$
<V_{x}>=\bar{V}_{x}+\tilde{v}_{x}
$$

where $\bar{V}_{x}$ is the time averaged velocity and $\tilde{v}_{x}$ is the oscillating velocity having a period equal to the wave period. The ensemble average has been evaluated by phase-averaging the velocity measurements. It is worth pointing out that in the acquired time series there was no evidence of spurious spikes, because the fluctuating velocities never exceed \pm 2 times the standard deviation of the measured component, therefore a pre-processing of the velocity time series was not needed. An example is shown in Fig. 2 where the three components of the acquired signal are shown (Fig. 2a) along with the decomposition of $V_{x}$ into the time averaged velocity $\bar{V}_{x}$, the oscillating velocity $\tilde{v_{x}}$ and the fluctuating component $v_{x}^{\prime}$ for a test case characterized by a depth averaged velocity $V_{D A}=0.045 \mathrm{~m} / \mathrm{s}$, an height $H=0.085 \mathrm{~m}$ and a period $T=1.6 \mathrm{~s}$ (Fig. $2 \mathrm{~b}$ ).

Part of the statistical analysis of the near bed flow reported in the following is carried out in terms of probability density function of the near bed velocity. This function provides unique information about the distribution of a given variable and can be used to compute all the relevant statistics of a turbulent flow. When a current interacts with orthogonal waves, as a result the probability density of the current velocity is modified by the waves. Knowledge of this interaction 
Table 2 Experimental conditions in the presend of a sandy bed (SB), gravel bed (GB) and rippled bed (RB). CO: current only; WO: wave only; WC: wave plus current.

Bottom roughness

\begin{tabular}{ccccccc}
\hline Type & $\begin{array}{c}V_{D A} \\
\left(\mathrm{~m}^{3} / \mathrm{s}\right)\end{array}$ & $\begin{array}{c}H \\
(\mathrm{~m})\end{array}$ & $\begin{array}{c}T \\
(\mathrm{~s})\end{array}$ & $\mathrm{SB}$ & $\mathrm{GB}$ & $\mathrm{RB}$ \\
\hline $\mathrm{CO}$ & 0.045 & - & - & $\mathrm{X}$ & $\mathrm{X}$ & $\mathrm{X}$ \\
$\mathrm{CO}$ & 0.067 & - & - & $\mathrm{X}$ & - & $\mathrm{X}$ \\
$\mathrm{CO}$ & 0.100 & - & - & $\mathrm{X}$ & $\mathrm{X}$ & $\mathrm{X}$ \\
WO & - & 0.085 & 0.80 & $\mathrm{X}$ & $\mathrm{X}$ & $\mathrm{X}$ \\
WO & - & 0.085 & 1.00 & $\mathrm{X}$ & $\mathrm{X}$ & $\mathrm{X}$ \\
WO & - & 0.085 & 1.20 & $\mathrm{X}$ & $\mathrm{X}$ & $\mathrm{X}$ \\
WO & - & 0.085 & 1.40 & $\mathrm{X}$ & $\mathrm{X}$ & $\mathrm{X}$ \\
WO & - & 0.085 & 1.60 & - & - & $\mathrm{X}$ \\
WO & - & 0.105 & 0.80 & $\mathrm{X}$ & $\mathrm{X}$ & $\mathrm{X}$ \\
WO & - & 0.105 & 1.00 & $\mathrm{X}$ & $\mathrm{X}$ & $\mathrm{X}$ \\
WO & - & 0.105 & 1.20 & $\mathrm{X}$ & $\mathrm{X}$ & $\mathrm{X}$ \\
WO & - & 0.105 & 1.40 & $\mathrm{X}$ & $\mathrm{X}$ & $\mathrm{X}$ \\
WO & - & 0.105 & 1.60 & - & - & $\mathrm{X}$ \\
WO & - & 0.120 & 1.00 & - & - & $\mathrm{X}$ \\
WC & 0.045 & 0.085 & 0.80 & $\mathrm{X}$ & $\mathrm{X}$ & $\mathrm{X}$ \\
WC & 0.045 & 0.085 & 1.00 & $\mathrm{X}$ & $\mathrm{X}$ & $\mathrm{X}$ \\
WC & 0.045 & 0.085 & 1.20 & $\mathrm{X}$ & $\mathrm{X}$ & $\mathrm{X}$ \\
WC & 0.045 & 0.085 & 1.40 & $\mathrm{X}$ & $\mathrm{X}$ & $\mathrm{X}$ \\
WC & 0.045 & 0.085 & 1.60 & $\mathrm{X}$ & $\mathrm{X}$ & $\mathrm{X}$ \\
WC & 0.045 & 0.105 & 0.80 & $\mathrm{X}$ & $\mathrm{X}$ & $\mathrm{X}$ \\
WC & 0.045 & 0.105 & 1.00 & $\mathrm{X}$ & $\mathrm{X}$ & $\mathrm{X}$ \\
WC & 0.045 & 0.105 & 1.20 & $\mathrm{X}$ & $\mathrm{X}$ & $\mathrm{X}$ \\
WC & 0.045 & 0.105 & 1.40 & $\mathrm{X}$ & $\mathrm{X}$ & $\mathrm{X}$ \\
WC & 0.045 & 0.105 & 1.60 & - & $\mathrm{X}$ & - \\
WC & 0.067 & 0.085 & 1.00 & $\mathrm{X}$ & $\mathrm{X}$ & $\mathrm{X}$ \\
WC & 0.100 & 0.105 & 0.80 & - & $\mathrm{X}$ & - \\
\hline
\end{tabular}
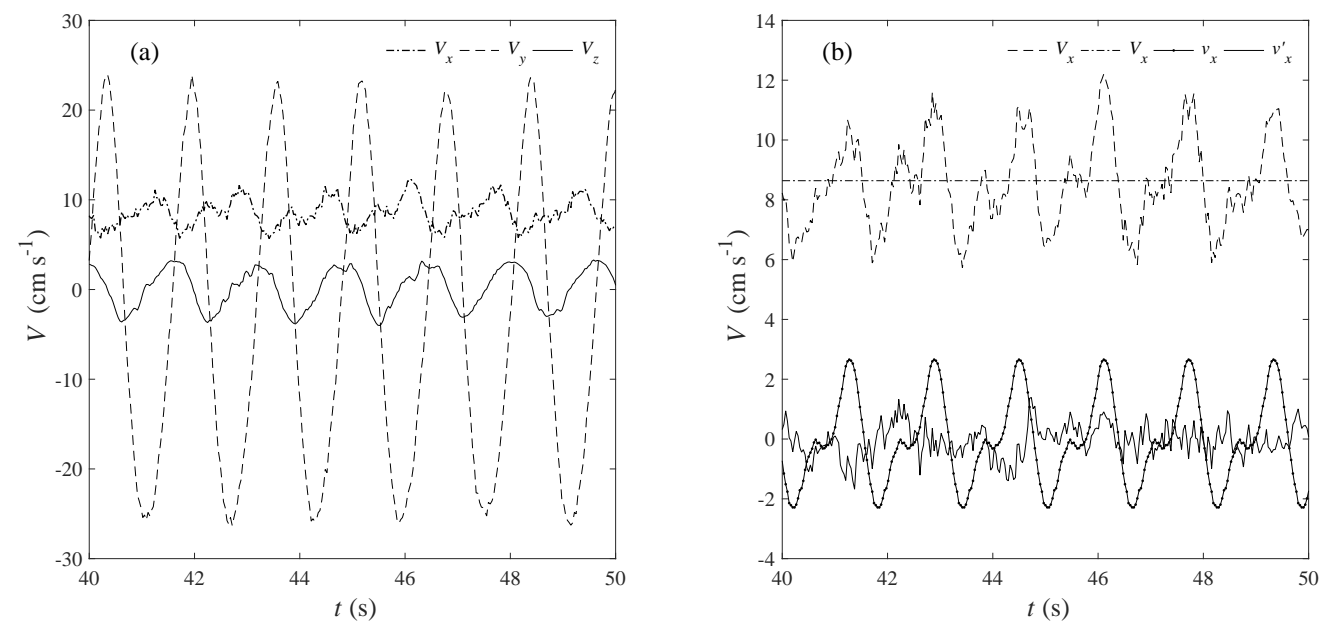

Figure 2 (a)Instantaneous value of the three velocity components in the three directions; (b) Decomposition of the instantaneous velocity $V_{x}$ into a time averaged velocity $\bar{V}_{x}$, an oscillating velocity $\tilde{v_{x}}$ and a fluctuating component $v_{x}^{\prime} . V_{D A}=0.045$ $\mathrm{m} / \mathrm{s} ; H=0.085 \mathrm{~m}, T=1.6 \mathrm{~s}$. 

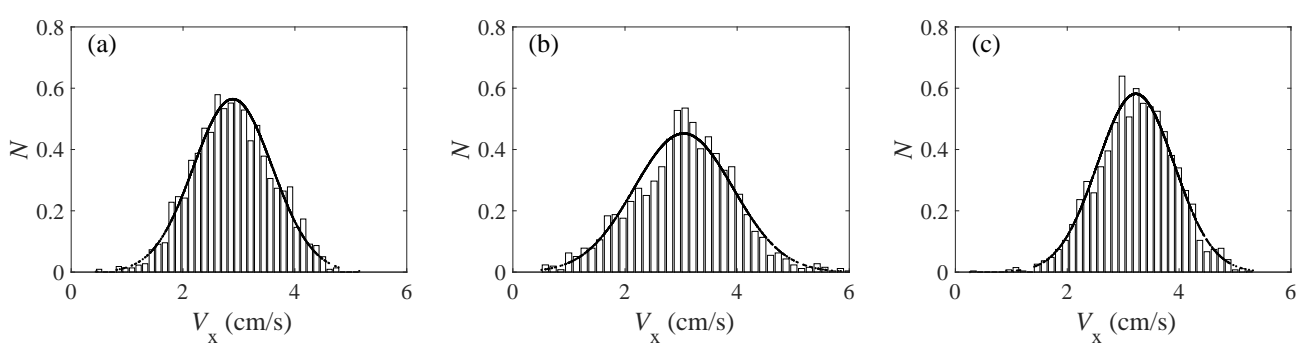

Figure 3 Frequency distribution of $\mathrm{V}_{x}$ in the current only case at a point $0.5 \mathrm{~cm}$ far from the bed; $V_{D A}=0.045 \mathrm{~m} / \mathrm{s}$ and different bed roughness: (a) SB; (b) GB; (c) RB (trough). A Gaussian PDF is also superimposed.

process and how it affects the probability density of the velocity fluctuations is relevant not only for the hydrodynamics but also for the sediment transport. Indeed wave-current interactions usually occur over a bed made up of loose materials that can be mobilized by an increase of the velocity fluctuations.

In Section 3.1 the frequency distribution of $V_{x}$ at a fixed location close to the bottom is analyzed. Section 3.2 is devoted to the analysis of the distribution of the turbulent component $v_{x}^{\prime}$, moreover the vertical profiles of the statistics for both the current only case and the wave plus current cases are inter-compared. Finally in Section 3.3 the analysis of the phase-averaged velocity component $V_{x}$ is presented.

\subsection{Statistical analysis of total near-bed velocity}

The statistics of the velocity component in the $\mathrm{x}$ direction have been analysed here. The frequency distribution of the bed velocity has been obtained by counting the number of occurrences $N *$ in $n$ classes of $V_{x}$ and by obtaining the relative number of occurrences $N$ as the ratio between $N *$ and the product between the class amplitude and the total number of occurrences. Here the number of classes $\mathrm{n}$ has been chosen equal to 40 , because such a value is sufficiently high to provide a good representation of the whole velocity distribution but still adequate to contain enough events in each class.

Figure 3 shows the probability distribution of one of the current only cases $\left(V_{D A}=0.045 \mathrm{~m} / \mathrm{s}\right)$ at the measuring point located closest to the bed, i.e. at about $0.5 \mathrm{~cm}$ above it; each plot corresponds to a different roughness condition, i.e. SB, GB and RB respectively. It can be observed that in the case of sandy bed the PDF is symmetric and the skewness is approximately zero. In the gravel and rippled bed cases the behaviour is rather similar to each other and the distributions negatively skew. This implies that the number of negative fluctuations is larger compared to that of the positive ones.

Similarly, in Fig. 4 the probability distributions for different currents $\left(V_{D A}=0.045 \mathrm{~m} / \mathrm{s}, 0.067\right.$ $\mathrm{m} / \mathrm{s}$ and $0.100 \mathrm{~m} / \mathrm{s}$ ) are shown. The distributions are all rather symmetric and closely follow the Gaussian PDF. As expected, as soon as the current velocity increases, the distribution becomes flatter and broader as a result of the increased turbulence.

When waves are added on the existing current, the flow shows a much more complex behaviour. In Figs 5, 6 and 7 the PDF of a SB, a GB and a RB roughness condition respectively for different wave plus current flows are reported. In all the figures the depth averaged velocity of the current is equal to $V_{D A}=0.045 \mathrm{~m} / \mathrm{s}$; this current has been superimposed to four wave conditions with two different wave periods (namely 0.8 and $1.4 \mathrm{~s}$ ) and two different wave heights $(0.085$ and 0.105 $\mathrm{m}$ ). The differences between the frequency distribution of the current velocity and the Gaussian probability density function increase as the period increases. In particular two main peaks are visible; in the rippled bed tests (Fig. 7), where the measurement point is located in the bedform trough and thus sheltered by the ripple lee side, such a feature turns out to be less apparent for 

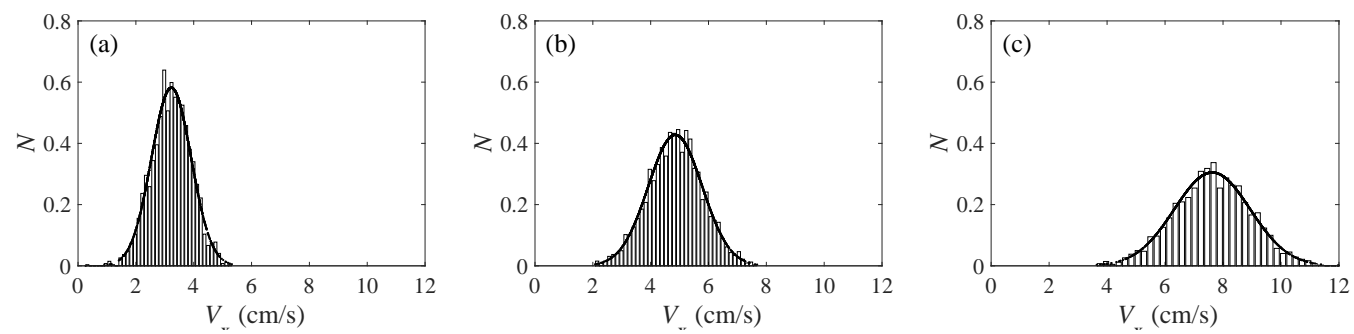

Figure 4 Frequency distribution of $\mathrm{V}_{x}$ in the current only case at a point $0.5 \mathrm{~cm}$ far from the bed; RB and different $V_{D A}$ (a) $0.045 \mathrm{~m} / \mathrm{s}$; (b) $0.067 \mathrm{~m} / \mathrm{s}$; (c) $0.100 \mathrm{~m} / \mathrm{s}$. A Gaussian PDF is also superimposed.

smaller wave heights. In general, the departure from the normal distribution increases with the wave period, while the wave height does not play a significant role. Similar features, even though not shown here for sake of brevity, have been observed also for intermediate periods $(T=1$ and 1.2 s).

As a matter of fact this feature is due to an oscillatory flow induced by waves also in the current direction. One of the main mechanisms through which the velocity in the current direction fluctuates is due to the advection of $x$-momentum along the vertical due to the vertical gradient of the mean current velocity. In the convective term of the Navier-Stokes equations, this advection is provided by the term $V_{z} \partial V_{x} / \partial z$ where $V_{z}$ is the vertical velocity which is largely due to the wave orbital motion. The effect of this term on the current velocity can be illustrated writing the equation $\partial V_{x} / \partial t=-V_{z} \partial V_{x} / \partial z$ which neglects several other terms. Although this equation represents only a rough approximation of the real phenomenon as it neglects several terms, it is sufficient for the purpose of a qualitative explanation of the oscillating behaviour of the current velocity. The right hand side of the previous equation is non-linear, therefore it is not easy to obtain rigorous information about the phase of oscillation induced by itself. If at first it is assumed that $\partial V_{x} / \partial z$ does not vary too much during the wave period and that it is positive, we observe that according to the previous equation the time derivative of the current velocity is positive when the vertical velocity is negative and vice-versa. The reasonableness of this result can be noted in Fig. 2 where the time development of the velocities along the three Cartesian axes is shown. It can be observed that most of the times when $V_{z}$ is positive the time derivative of the current velocity is negative and viceversa. Thus if the vertical velocity oscillates like $\sin (\sigma t)$ the current velocity oscillates as $\cos (\sigma t)$. This process occurs along the entire water column, however due to the exponential orbital velocity decay over the vertical, it is more apparent only for larger waves, whose effect penetrates up to the bottom. Of course the neglected terms in the previous equation and the non-linear effect due to the time variation of the vertical gradient of the current velocity, make the picture a little different from that described above. Another effect contributing to the oscillating behaviour of the current velocity is the time variation of the water depth. In particular, under the wave crest the total water depth is larger, inducing a decrease of velocity with respect to its mean value. On the opposite, under the wave through the total water depth is smaller, resulting into an increase of the velocity and a consequent peak at $V_{x}$ larger than the mean value.

The two peaks of the PDF that can be observed in Figs 5-7 (b-d) are made up by velocities occurring around the maximum and the minimum of the oscillating current velocity (as shown in Fig. 2). This result is similar to that occurring with a sinusoidal function which has a PDF with two peaks of the same magnitude, one peak is located at the maximum and the other at the minimum. In the present case however the shape of the function is not exactly a sinusoidal, thus the two peaks of the PDF are different.

According to the previously mentioned processes, the $V_{x}$ data have been split into two classes of current velocities, the first corresponding to positive values of the wave velocity component (i.e. to the passage of a wave crest, or onshore directed wave velocities $V_{y}$ ) hereinafter referred 

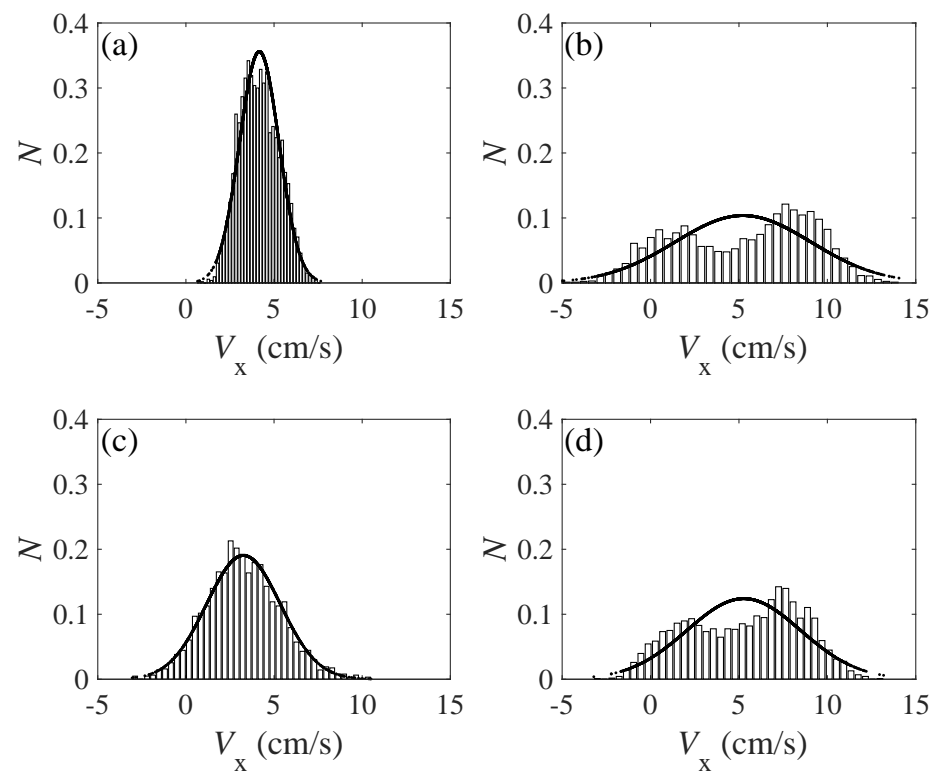

Figure 5 Frequency distribution of $V_{x}$ in the wave plus current case at a point $0.5 \mathrm{~cm}$ far from the bed for SB bed roughness, $V_{D A}=0.045 \mathrm{~m} / \mathrm{s}$ and different wave conditions: (a) $H=0.085 \mathrm{~m}, T=0.8 \mathrm{~s}$; (b) $H=0.085 \mathrm{~m}, T=1.4 \mathrm{~s} ;(\mathrm{c}) H=0.105 \mathrm{~m}, T=0.8$ s; (d) $H=0.105 \mathrm{~m}, T=1.4 \mathrm{~s}$. A Gaussian PDF is also superimposed.

to as $V_{x}^{y+}$, the second corresponding to negative values of the wave velocity component (i.e. to the trough passage, or offshore directed wave velocities $V_{y}$ ) hereinafter referred to as $V_{x}^{y-}$. The same decoupling procedure can also be applied in order to obtain the quantities $V_{x}^{z+}$ and $V_{x}^{z-}$, corresponding respectively to current velocities occurring simultaneously to a positive (upward) or negative (downward) vertical velocity. Several probability density functions have been determined for the two classes of data.

The PDFs of $V_{x}^{y+}$ and $V_{x}^{y-}$ are shown in Fig. 8 for $V_{D A}=0.045 \mathrm{~m} / \mathrm{s}, H=0.085 \mathrm{~m}$ and $T=1.2 \mathrm{~s}$. It is possible to observe that for all the roughness conditions, $V_{x}^{y+}$ and $V_{x}^{y-}$ distributions are rather separated one from the other, and the peak of the PDF of $V_{x}^{y+}$ always occurs at smaller velocities than that of $V_{x}^{y-}$.

Indeed, as above mentioned, in the presence of the combined wave-current flow, according to the mass conservation principle, velocity in the current direction should be decreased under the wave crest and increased under the wave trough. Hence, the peak of the $V_{x}$ velocity should be $180^{\circ}$ out of phase compared to $V_{y}$, while, due to vertical advection of x-momentum described by the term $V_{z} \partial V_{x} / \partial z$ in the Navier-Stokes equations a phase shift could occur. Assuming that the effects of the continuity are more significant than the others, the decoupling by using the sign of $V_{y}$ should be the most significant one. However, as a result of the above effects, the oscillation of $V_{x}$ may be phase-shifted with respect to $V_{y}$. It follows that the separation of the peaks of the pdf at higher or lower velocities is not exact as shown in Fig. 8a and b, for the SB and GB conditions. In the presence of the rippled bed, the boundary layer separates at the ripple crest by generating a vortex shedding. It should be noted that since the ripple length is $12.5 \mathrm{~cm}$, the spatial scale of such vortices has an order of magnitude similar to that of the water depth $(D=30 \mathrm{~cm})$. It follows that the transfer of momentum induced by the vortices increases the randomness of $V_{x}$ such that the harmonic component with period equal to that of the waves, which is mostly responsible for the double peaks, becomes not much larger than the others. This explain the reason why the PDF of $V_{x}$ over a ripple bed in some cases does not show double peaks, as it can be observed in Fig. $7 \mathrm{~b}$.

Moreover, as for the current only case, the PDF is rather flat for the GB case and sharper for SB 

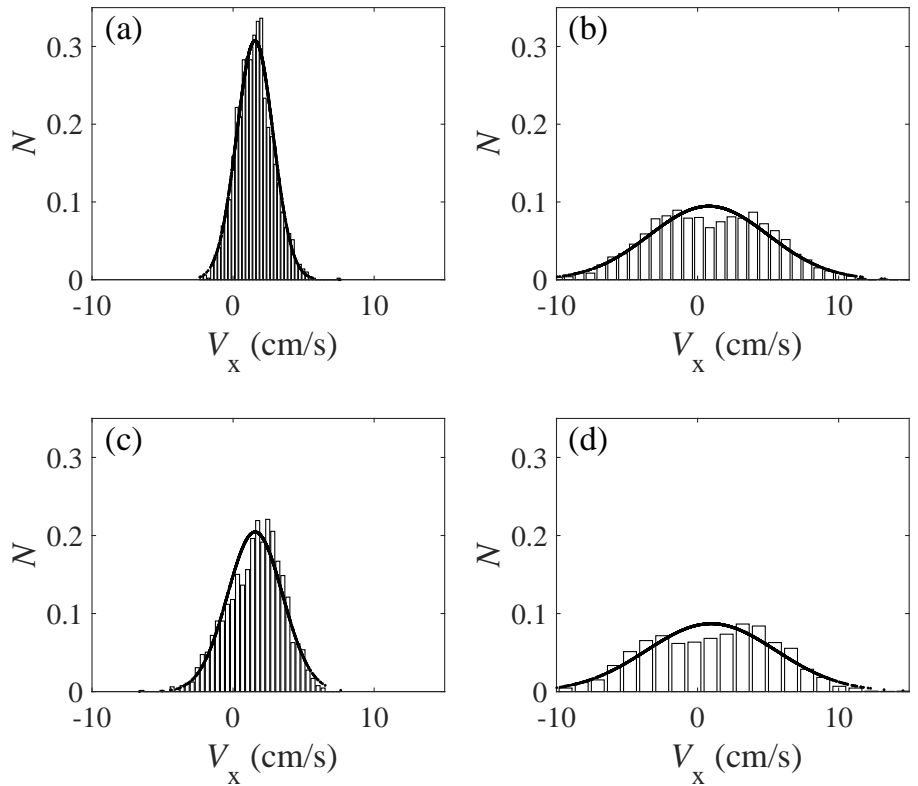

Figure 6 Frequency distribution of $V_{x}$ in the wave plus current case at a point $0.5 \mathrm{~cm}$ far from the bed for GB bed roughness, $V_{D A}=0.045 \mathrm{~m} / \mathrm{s}$ and different wave conditions: (a) $H=0.085 \mathrm{~m}, T=0.8 \mathrm{~s}$; (b) $H=0.085 \mathrm{~m}, T=1.4 \mathrm{~s}$; (c) $H=0.105$ $\mathrm{m}, T=0.8 \mathrm{~s}$; (d) $H=0.105 \mathrm{~m}, T=1.4 \mathrm{~s}$. A Gaussian PDF is also superimposed.
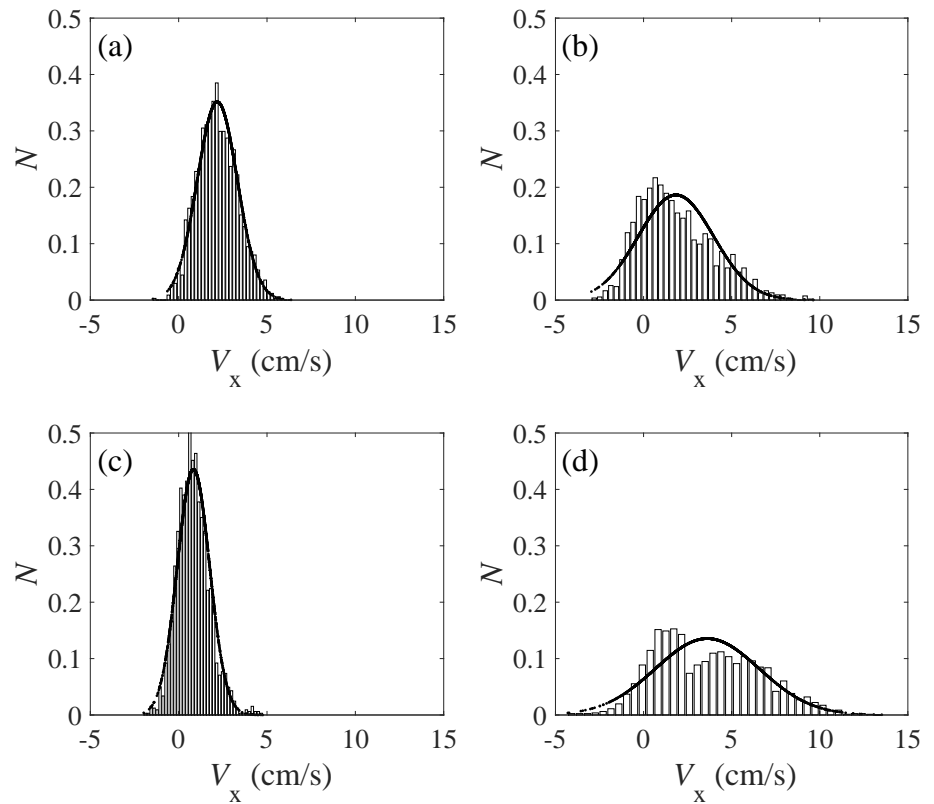

Figure 7 Frequency distribution of $\mathrm{V}_{x}$ in the wave plus current case at a point $0.5 \mathrm{~cm}$ far from the bed for RB bed roughness, $V_{D A}=0.045 \mathrm{~m} / \mathrm{s}$ and different wave conditions: (a) $\mathrm{H}=0.085 \mathrm{~m}, \mathrm{~T}=0.8 \mathrm{~s}$; (b) $\mathrm{H}=0.085 \mathrm{~m}, \mathrm{~T}=1.4 \mathrm{~s}$; (c) $\mathrm{H}=0.105$ $\mathrm{m}, \mathrm{T}=0.8 \mathrm{~s} ;$ (d) $\mathrm{H}=0.105 \mathrm{~m}, \mathrm{~T}=1.4 \mathrm{~s}$. A Gaussian PDF is also superimposed. 

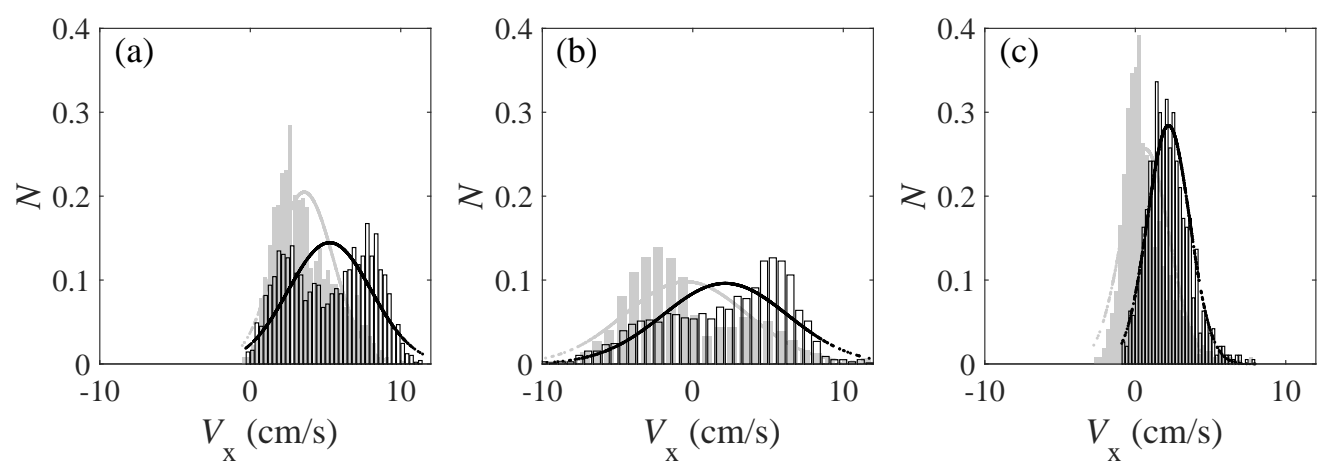

Figure 8 Frequency distribution of $V_{x}$ in the wave plus current case at a point $0.5 \mathrm{~cm}$ far from the bed for $V_{D A}=0.045$ $\mathrm{m} / \mathrm{s}, H=0.085 \mathrm{~m}, T=1.2 \mathrm{~s}$, and the following bed roughness: (a) SB; (b) GB; (c) RB (trough). Black and white bar plot: current velocities acquired during the passage of negative wave velocities $\left(V_{x}^{y-}\right)$. Grey bar plot: current velocities acquired during the passage of positive wave velocities $\left(V_{x}^{y+}\right)$. The corresponding Gaussian PDFs are also superimposed.

and RB tests, where the standard deviation is smaller. This difference is a macroroughness effect and can be attributed to the fact that the gravels are larger and randomly positioned onto the bed. This affects the level of turbulence within the boundary layer, by spreading the near-bed velocities over a wide range of values.

In Fig. 9a the separation distance between the two peaks of the PDF is plotted against the wave period $T$ for the SB case. In the plot data characterized by two different wave heights are shown. Such a distance has been evaluated as the difference between the mean values of the two Gaussian PDFs that describe the two classes of current velocities, the first corresponding to positive values of the wave velocity component, the second corresponding to negative values of the wave velocity component, as it will be better detailed in the following. As mentioned, such a distance increases for increasing $T$. As a matter of fact, the presence of the double-peak distribution indicates the existence of an oscillating flow, induced by the presence of the waves, also in the current direction. As a consequence of this, the distance between the two peaks $\Delta V_{x}^{y}$ is larger for higher wave heights.

The standard deviation of the two different PDF $\sigma V_{x}^{y+}$ and $\sigma V_{x}^{y-}$, obtained as described above, is plotted in Fig. 9b, as a function of the wave period. The results indicate that the standard deviation tends to increase with the wave period as well. Such an increase seems to be independent both on the wave phase and on the intensity of the current, demonstrating that as the wave orbital motion is more intense at the bed, the fluctuations of the current velocity increase in the same way both under the wave crests and the wave troughs. It turns out that shallower water waves should play a major role on the increase of turbulent fluctuations at the bed.

In Fig. 10 and 11 the attention is focused on the rippled bed case: the previously described procedure has been applied to determine the PDF of $V_{x}^{y+}$ and $V_{x}^{y-}$ and the PDF of $V_{x}^{z+}$ and $V_{x}^{z-}$ respectively. In Fig. 10(a) and 11(a) the measurement point is located at an intermediate position between the offshore ripple crest and the trough (point 2 with reference to the sketch in Fig. 1); in 10(b) and 11(b) the point is located at the trough (point 3), then in Fig. 10(c) and 11(c) the second intermediate point is considered (point 4) and finally Fig. 10(d) and 11(d) report measurements gathered at the onshore crest (point 5). Only the onshore crest has been plotted as the results obtained at points 1 and 5 are in good agreement.

Looking at these two figures, at first glance one may notice that the behaviour of $V_{x}^{y+}$ and $V_{x}^{z+}$ as well as the one of $V_{x}^{y-}$ and $V_{x}^{z-}$ is slightly similar. In particular, at the first intermediate point (point 2, Fig. 10(a) and 11(a)) the two distributions are unexpectedly roughly superimposable. This evidence has been related to the vorticity dynamics at the rippled bed. Indeed, as shown by Faraci et al. (2008), under the wave crest, the leeward side is affected by a high clockwise vorticity generated by the vortex shedding at the ripple crest. It follows that water particles coming from 

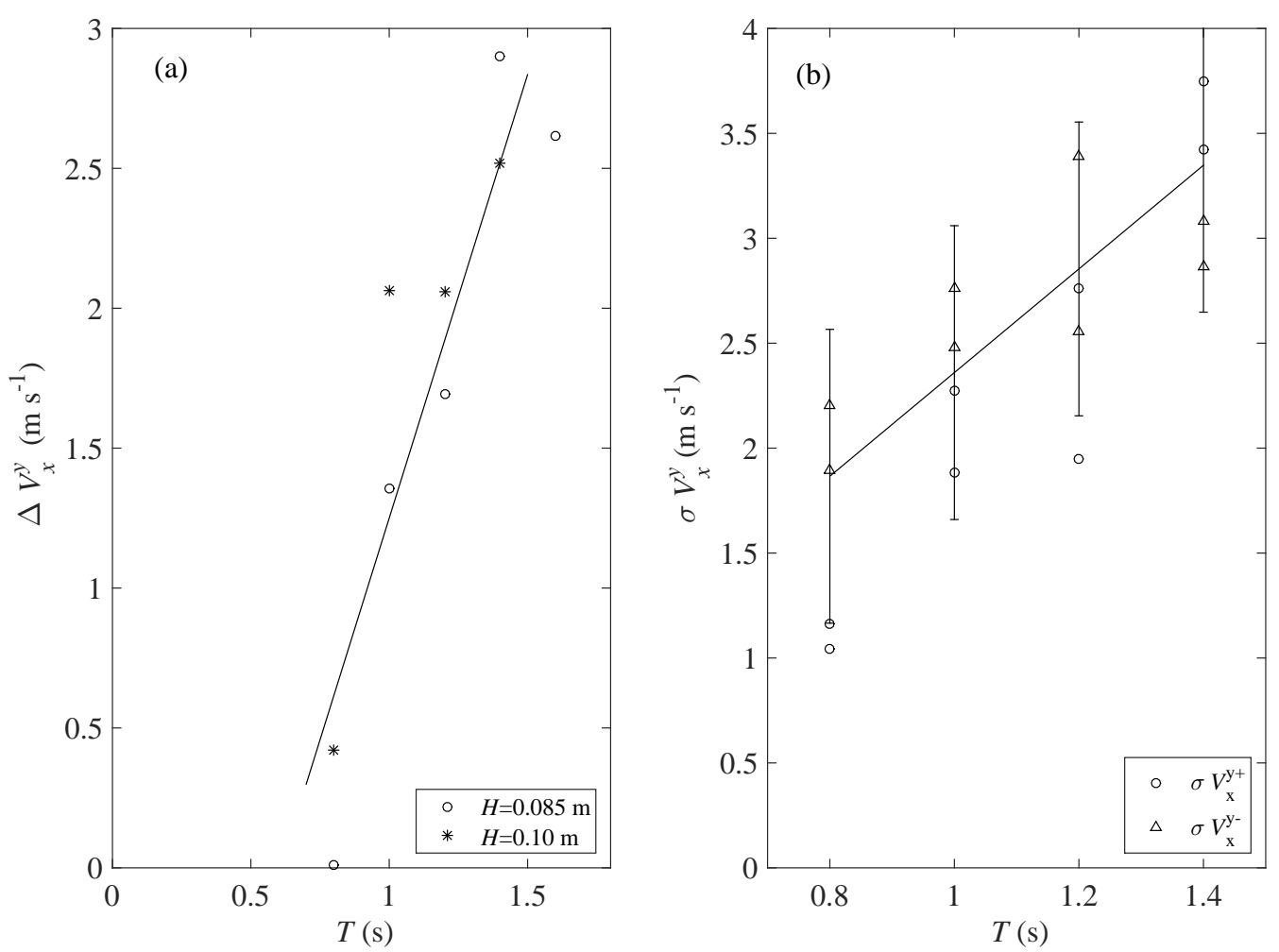

Figure 9 (a)Variation of $\Delta \mathrm{V}_{x}^{y}$ as a function of $T$; (b)Variation of $\sigma \mathrm{V}_{x}^{y}$ as a function of $T$. SB tests for $V_{D A}=0.045 \mathrm{~m} / \mathrm{s}$.

the accelerated seaward side are pulled down in the leeward side by the vortex generated by flow separation. Since these fluid particles come from a region farther from the bed, where the velocity is large, around the crest phase an increase of $V_{x}$ occurs which partially compensates the decrease of the velocity determined by the crest passage as described also in section 3.3.

Therefore this mechanism leads to level the values of $V_{x}^{y+}$ and $V_{x}^{y-}$ (or $V_{x}^{z+}$ and $V_{x}^{z-}$ ).

Similarly, at the second intermediate point (point 4, Fig. 10(c) and 11(c)), under the wave trough, high vorticity pulls down the fluid coming from higher velocity regions, leading to an increase of $V_{x}^{y-}$ with respect to $V_{x}^{y+}$, thus at point 4 the two distributions are more separated than at point 2. Both ripple crest and trough are less affected by such a mechanism as there the vorticity is smaller.

Under the wave crest, at all the ripple locations, the current velocities are more peaked around the mean value than under the wave trough. This depends on the wave shape, which is indeed characterized by narrow and high crests and flat and broad troughs, which last more than one half period. Such evidence is also confirmed by observing that the sum of occurrences of $V_{x}^{y-}$ is always larger than that of $V_{x}^{y+}$.

In Fig. 12 the modification of the mean flow direction as a consequence of the wave superposition on the existing current is shown. For the sake of completeness the mean flow is also shown here, even though the results have been already discussed in Musumeci et al. (2006) and in Faraci et al. (2008). Figure 12(a) and (d) concern the SB case, Fig. 12(b) and (e) the GB case, and Fig. 12(c) and (f) the RB case. The angle $\theta=0^{\circ}$ corresponds to the current alone propagation direction (see Fig. 1c). When waves are added to the current, in the case of SB roughness the mean angle veers of about $-10^{\circ}$, as a consequence of the undertow current resulting from the wave propagation. This result is in very good agreement with that found by Lim and Madsen (2016). When a rippled bed or a gravel bed is considered, the veering shows different features. Indeed close to the bed the 

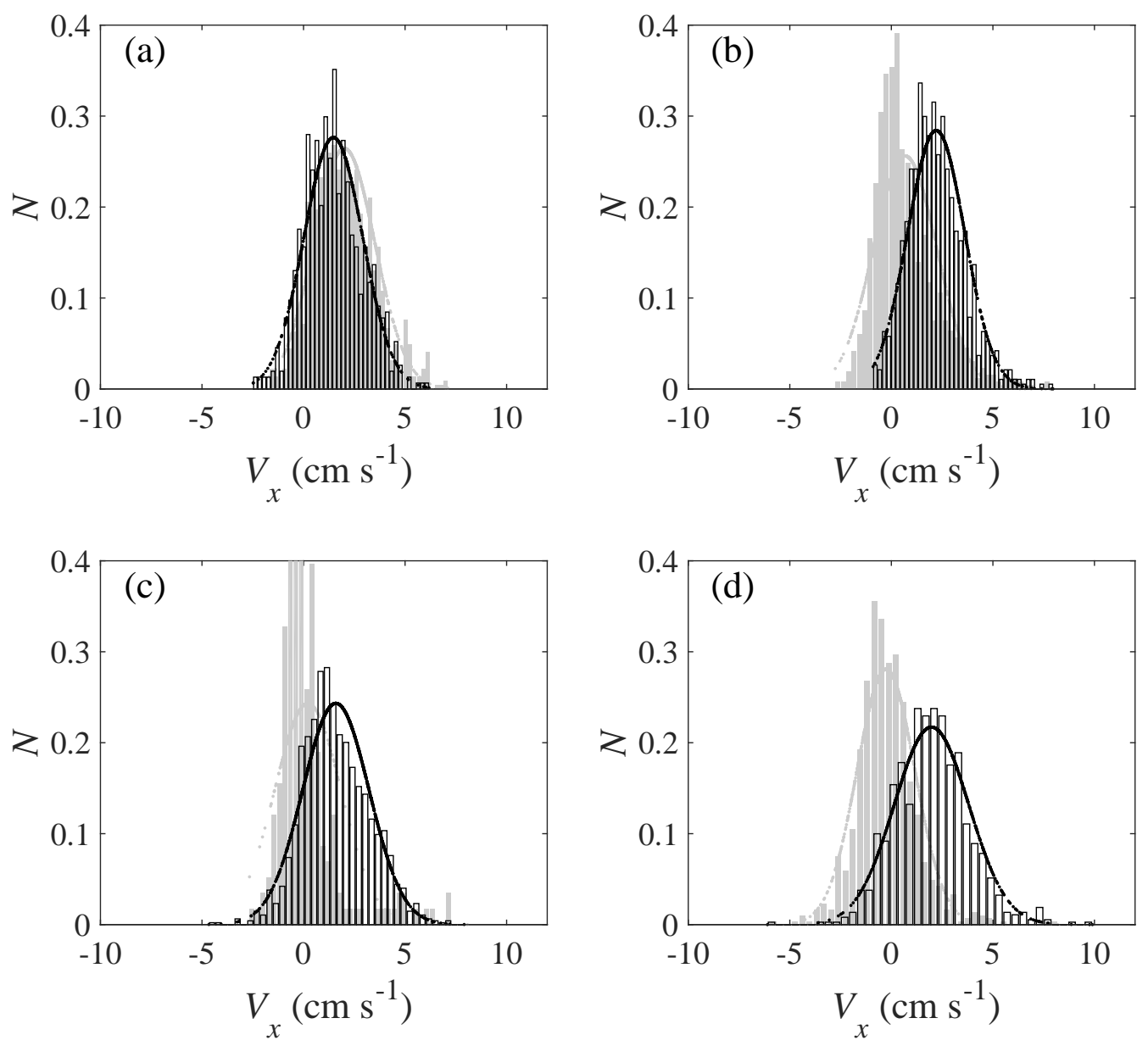

Figure 10 Frequency distribution of $V_{x}^{y+}$ and $V_{x}^{y-}$ for the rippled bed case: (a) intermediate between crest and trough; (b) trough; (c) intermediate between trough and crest; (d) crest. The measuring point is located $0.5 \mathrm{~cm}$ far from the bottom. Same test conditions and symbols as in Fig. 8. Black and white bar plot: current velocities acquired during the passage of negative wave velocities $\left(V_{x}^{y-}\right)$. Grey bar plot: current velocities acquired during the passage of positive wave velocities $\left(V_{x}^{y+}\right)$. The corresponding Gaussian PDFs are also superimposed.

current veers towards the beach up to $5^{\circ}$, then $\theta$ decreases quite abruptly up to $-25^{\circ}$ at about $z=2 \mathrm{~cm}$. At this depth the local effects due to the roughness are transferred onto the mean flow, with an overall increase of the veering angle. Lim and Madsen (2016) interpret such a process as a consequence of turbulence asymmetry and mean momentum transfer. The decrease of the veering angle in the GB and RB cases is due to the setup of an offshore directed streaming near the bed, generated by the asymmetry in the wave shape and the large turbulent fluctuations induced by the large roughness that characterizes these two cases (Scandura, Faraci, \& Foti, 2016). In the upper part of the measured water column in the SB case the veering angle tends to increase due to the closeness of the water surface, as also observed in Lim and Madsen (2016). In the GB and RB cases the opposite occurs: indeed $\theta$ tends to line up to $\approx-18^{\circ}$. The results of Lim and Madsen (2016) in the case of rough bed are in substantial agreement with the present findings. However it is worth pointing out that they do not observe the inversion of veering angle close to the bed probably because they did not measure within the $2 \mathrm{~cm}$ closest to the bed. 

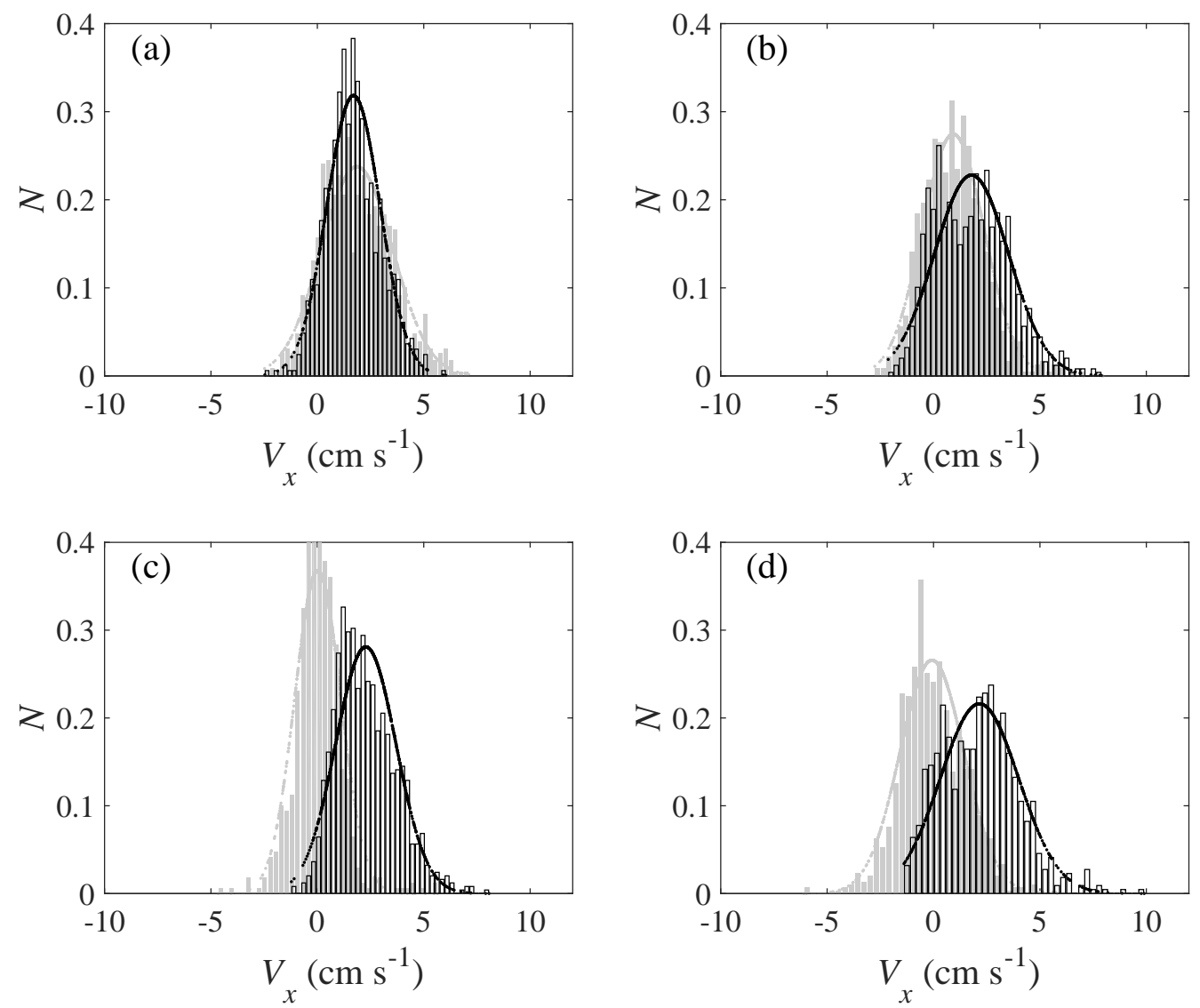

Figure 11 Frequency distribution of $V_{x}^{z+}$ and $V_{x}^{z-}$ for the rippled bed case: (a) intermediate between crest and trough; (b) trough; (c) intermediate between trough and crest; (d) crest. The measuring point is located $0.5 \mathrm{~cm}$ far from the bottom. Experimental test conditions as in Fig. 8 Black and white bar plot: current velocities acquired during the passage of negative vertical velocities $\left(V_{x}^{z-}\right)$. Grey bar plot: current velocities acquired during the passage of positive vertical velocities $\left(V_{x}^{z+}\right)$. The corresponding Gaussian PDFs are also superimposed.

\subsection{Statistical analysis of turbulent fluctuations}

The statistics of the turbulent fluctuations $v_{x}^{\prime}$, as described in equation (1), are presented here. Figure 13 shows the probability distribution for the wave plus current condition characterized by $V_{D A}=0.045 \mathrm{~m} / \mathrm{s}, H=0.105 \mathrm{~m} ; T=1.2 \mathrm{~s}$, for the three bottom roughness cases SB, GB and RB respectively. It can be noted that such a component behaves differently from $V_{x}$. Indeed in the PDF there is no evidence of double peaks. This is mainly due to the fact that $v_{x}^{\prime}$ does not contain the fluctating velocity induced by the wave motion although this motion affects the turbulent component as it will be shown in the following. Figure 13 also shows that the probability distribution is rather well described by the Gaussian distribution especially for sand and rippled bed cases. It can be noted that the largest turbulent fluctuations are of about $5 \mathrm{~cm} / \mathrm{s}$ which are higher than the current depth averaged velocity.

A measure of the width of the probability distribution is provided by the standard deviation of the velocity fluctuations, defined as $\overline{\left(v_{x}^{\prime 2}\right)^{1 / 2}}$. Figure 14 (a) and (b) shows the vertical profiles of the standard deviation for the current only tests shown in Fig. 3 and 4 respectively.

In Fig. 14a the standard deviation for the sand bed case is larger than in the ripple bed case but the largest values are obtained for the gravel bed because of the large element roughness. Although 

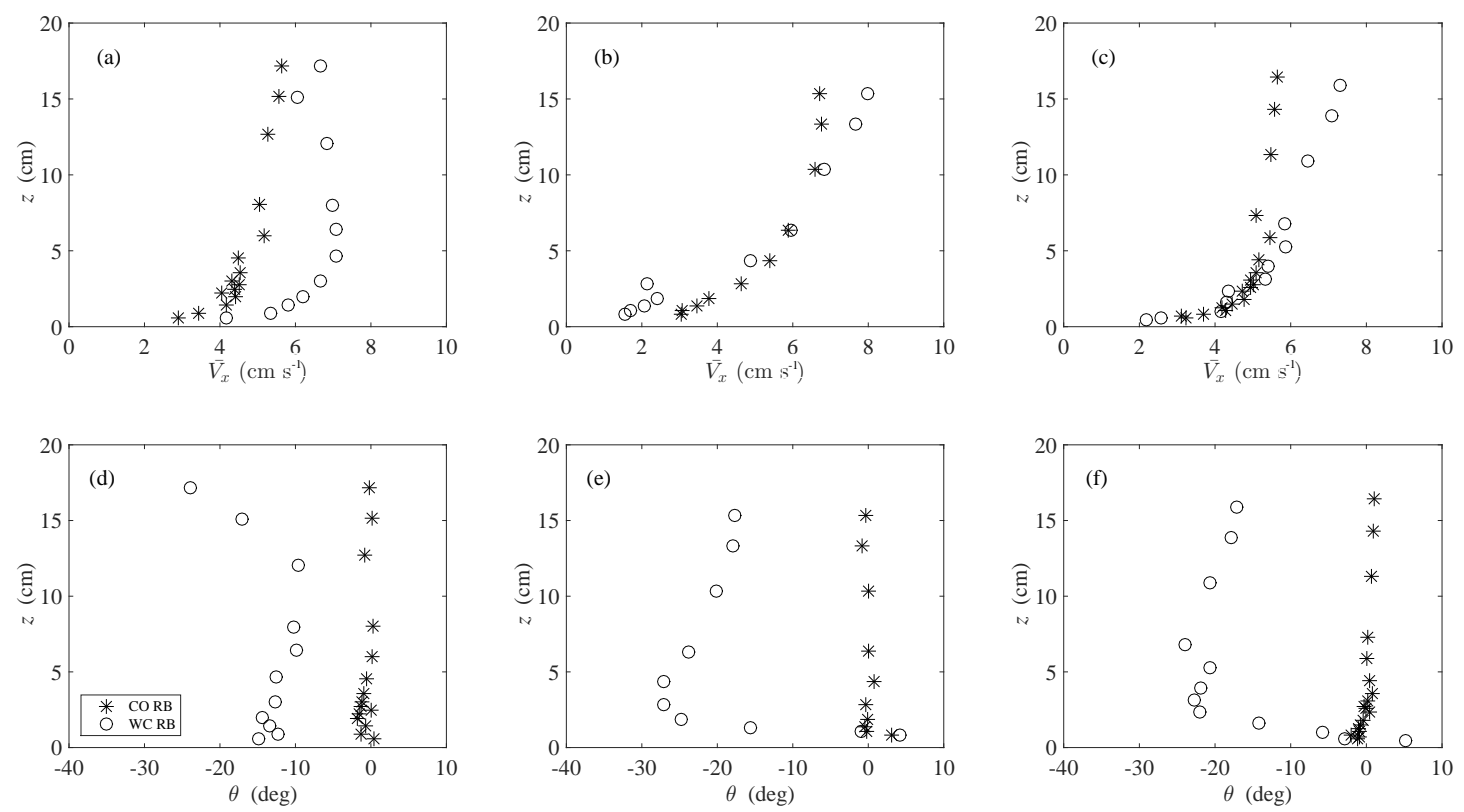

Figure 12 Mean flow $(\mathrm{a}, \mathrm{b}, \mathrm{c})$ and angle of mean flow $(\mathrm{d}, \mathrm{e}, \mathrm{f})$ for current alone and wave plus current flow for different roughness conditions. $V_{D A}=0.045 \mathrm{~m} / \mathrm{s} ; H=0.085 \mathrm{~m} ; T=0.8 \mathrm{~s} .(\mathrm{a}, \mathrm{d}) \mathrm{SB} ;(\mathrm{b}, \mathrm{e}) \mathrm{GB} ;(\mathrm{c}, \mathrm{f}) \mathrm{RB}$.
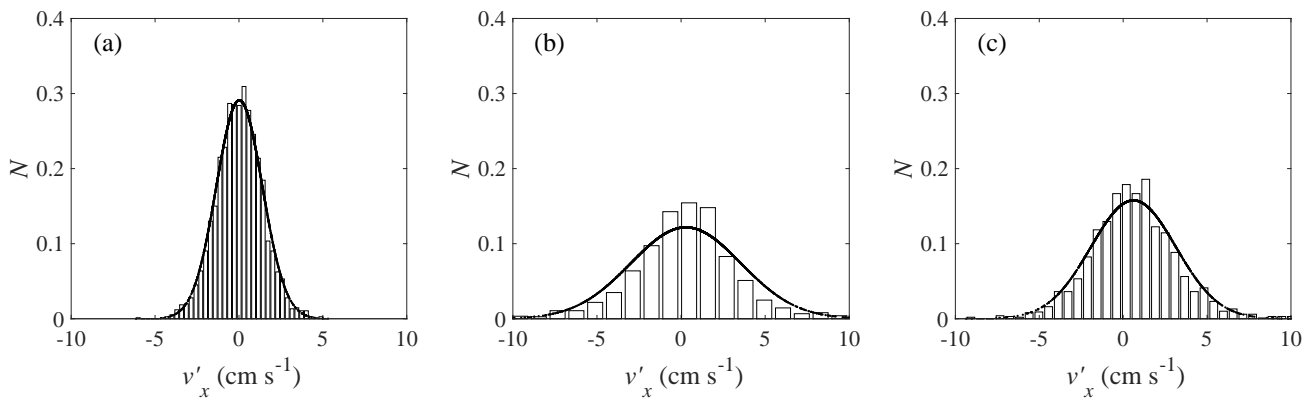

Figure 13 Frequency distribution of turbulent fluctuations $v_{x}^{\prime}$ in the wave plus current case at a point $0.5 \mathrm{~cm}$ far from the bed; $V_{D A}=0.045 \mathrm{~m} / \mathrm{s} ; H=0.105 \mathrm{~m} ; T=1.2 \mathrm{~s}$ and different bed roughness: (a) SB; (b) GB; (c) RB (trough). A Gaussian PDF is also superimposed.

in the RB case the roughness is also large, since the current flows parallel to the ripple crests, it does not significantly affect the turbulence intensity. The vertical distribution of the standard deviation shows that turbulence is much intense close to the bed. It decreases with $z / D$ up to a certain elevation $z^{*}$ beyond which it takes rather constant values. In particular, in Fig. 14a it can be observed that $z^{*}$ is equal to about $0.2-0.25$ for both the SB and the RB tests, whereas for the gravel bed case $z^{*}$ is larger $\left(z^{*}=0.35\right)$ because the large random roughness of the gravel bed (GB) produces an intense turbulence which extends over a larger portion of the water column.

For the ripple bed case shown in Fig. 14b the effect on the standard deviation of varying the current velocity is also shown. Even in this case values are higher close to the bed and then tend to reach a constant value of about $0.6,0.75$ and 0.9 for $V_{D A}$ respectively equal to $0.045 \mathrm{~m} / \mathrm{s}, 0.067$ $\mathrm{m} / \mathrm{s}$ and $0.100 \mathrm{~m} / \mathrm{s}$. However, the level beyond which such values are achieved remains similar to that of the previous case $\left(z^{*}=0.25\right)$.

Figure 14c shows the standard deviation for waves plus current conditions having current char- 

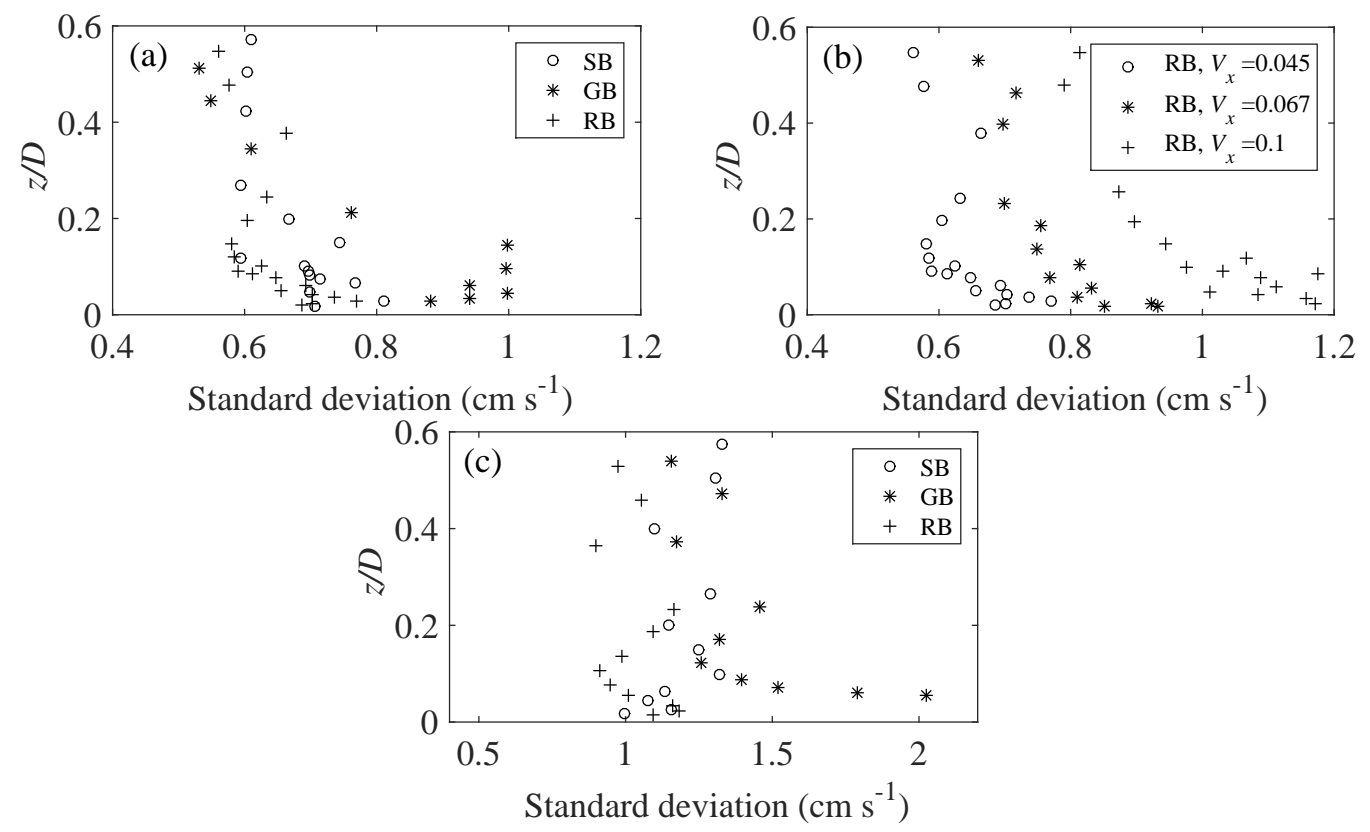

Figure 14 Vertical profile of the standard deviation of $V_{x}$ for (a) test cases as in Fig. 3; (b) RB roughness condition and different $V_{D A}$ as in Fig. 4; (c) different roughness and waves plus currents as in Fig. 8.

acteristics equal to those of Fig. 14 (a) and a wave with $H=0.085 \mathrm{~m} / \mathrm{s}$ and $T=1.2 \mathrm{~s}$. Adding an orthogonal wave to a current causes a large increase of the standard deviation of the turbulent fluctuations. Close to the bottom, for both ripple and sand bed cases, the standard deviation increases from about 0.8 for the current only case up to about 1.2 for the waves plus current. For the gravel bed, close to the bottom the standard deviation doubles the value due to the current only.

Figure 15 shows the trend of the Reynolds stress $\overline{v_{x}^{\prime} v_{z}^{\prime}}$ along the depth for the current only condition characterized by $V_{D A}=0.045 \mathrm{~m} / \mathrm{s}$ and for the wave plus current condition obtained by superimposing a wave having $H=0.085 \mathrm{~m}$ and $T=1.2 \mathrm{~s}$ on the previous current and considering the three different types of bed. Near the bottom the magnitude of the Reynolds stress for the gravel bed is much larger than that of the other two types of bed roughness. On the other hand the magnitudes of the Reynolds stress of the SB and of the RB cases are close to each other, but that of the sand is slightly larger than the other one. In the wave plus current case (Fig. 15b) the magnitude of the Reynolds stress increases with respect to the current only case. Generally it is larger for the gravel bed case except near the bed where the Reynolds stress is similar for all the three cases. It is mostly negative along the depth as it generally occurs for a steady turbulent current. In the current only case it reaches its minimum value at an elevation $z / D \approx 0.08$ for all the roughness conditions, while the minimum value changes depending on the bed condition. In the wave plus current case its value close to the bed is similar to the current only case, while it further decreases at an elevation $z / D \approx 0.2$. However, in the upper part of the water column for all the conditions the Reynolds stress tends to increase and for the sand bed case it become even positive.

In Fig. 16 the skewness $S=v_{x}^{\prime 3} /\left(\overline{v_{x}^{\prime 2}}\right)^{3 / 2}$ and the flatness $F=v_{x}^{\prime 4} /\left(\overline{v_{x}^{\prime 2}}\right)^{2}$ factors of the current velocity are shown versus the bottom distance, made non-dimensional by means of the water depth $D$. The skewness is a measure of the asymmetry of the probability density function with respect to the average value $\overline{V_{x}}$. For symmetric distributions with respect to $\overline{V_{x}}$, the skewness vanishes; an example of that behaviour is represented by the normal distribution. When the skewness is negative the largest fluctuations of $v_{x}^{\prime}$ are negative; the opposite occurs if the skewness is positive. The flatness provides a measure of the peakedness of the probability density function. In particular, 

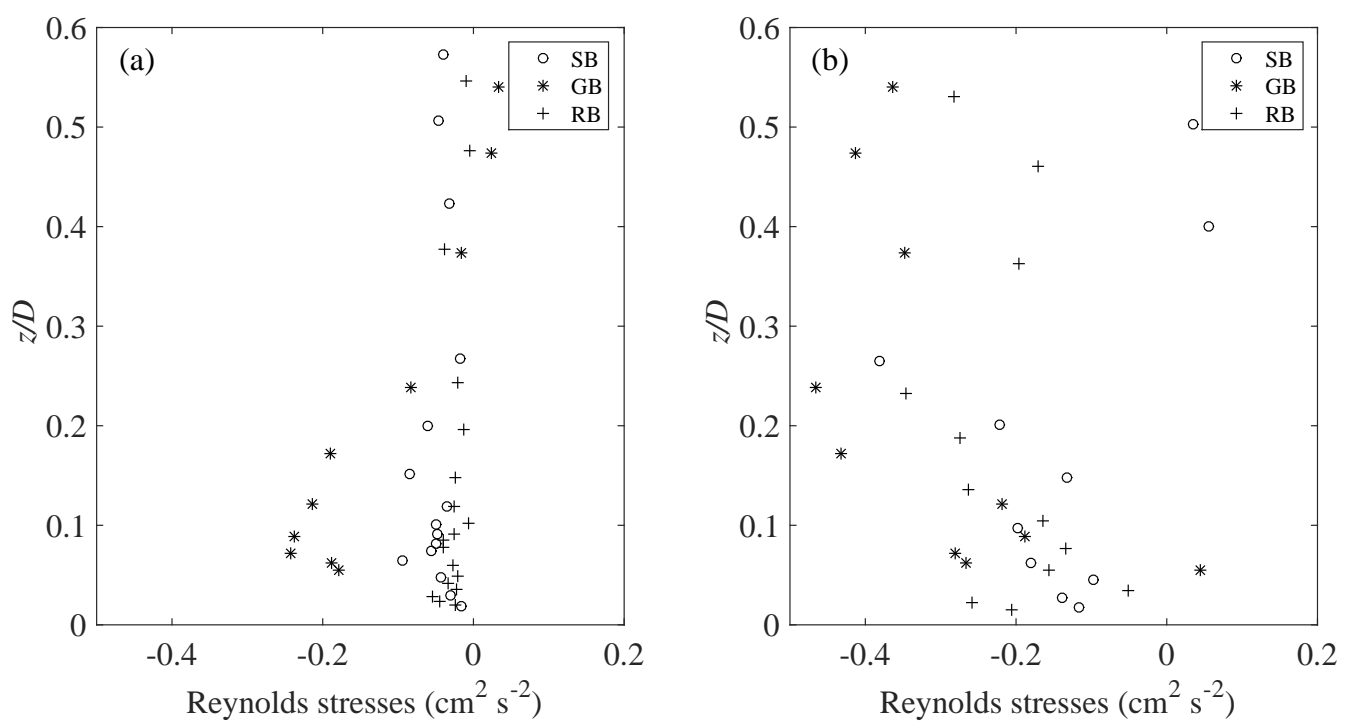

Figure 15 Reynolds stresses of: (a) current only flow and (b) wave plus current flow. $V_{D A}=0.045 \mathrm{~m} / \mathrm{s} ; H=0.085 \mathrm{~m} ; T=1.2$
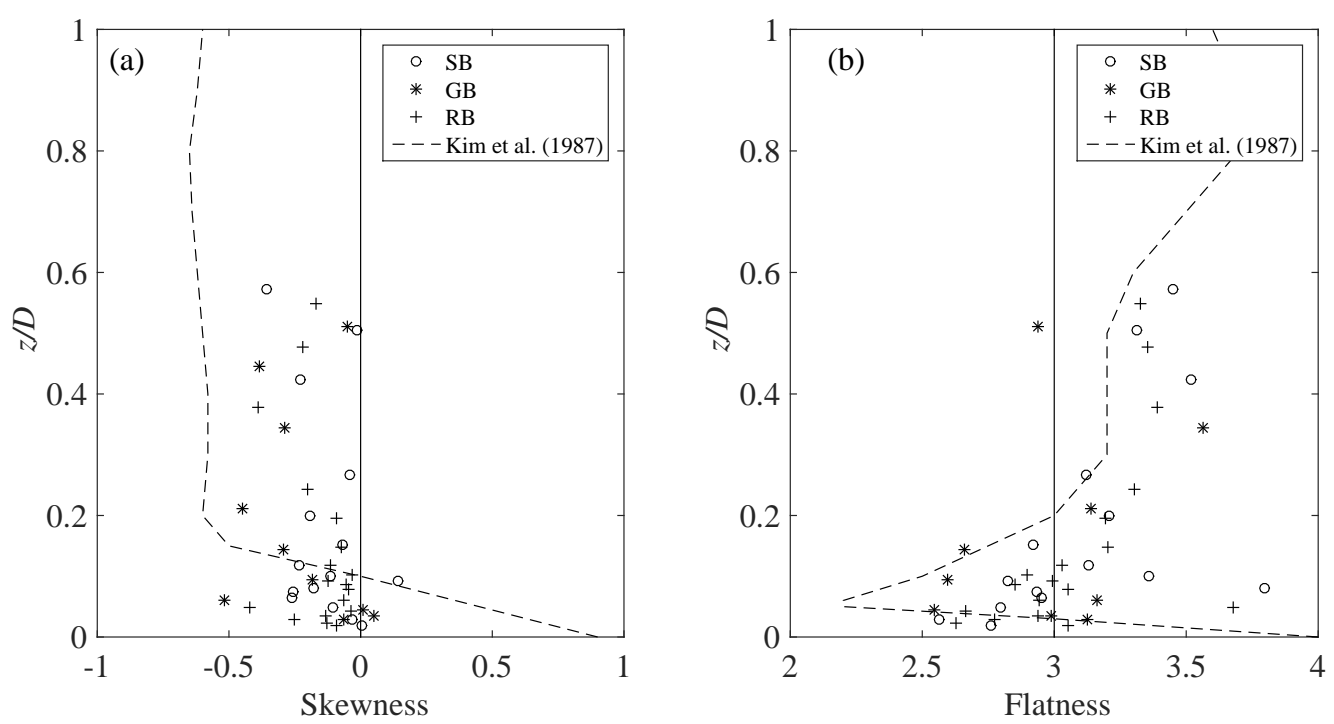

Figure 16 Vertical profile of skewness and flatness factors for SB, GB and RB (trough) beds. Experimental conditions as in Fig. 3.

the flatness takes the value of 3 in the case of a normal distribution. In the present analysis, in the current only case the skewness has small values very close to the bed, then it decreases reaching values of -0.3 to -0.4 far from the bed. The flatness assumes values close to 3 near the bed, then it falls below 3 for $0.02<z / D<0.15$ and finally it increases attaining values of about 3.5. These results show that a region close to the bottom exists where the skewness is small and the flatness is close to 3, thus explaining why in Fig. 13 the frequency distribution is well described by a Gaussian distribution.

Similar results were also numerically found by Kim et al. (1987) for a turbulent channel flow at low Reynolds numbers. These results are also superimposed on both graphs, showing a trend similar to the present ones for the different kinds of roughness. A similar analysis was also performed by 

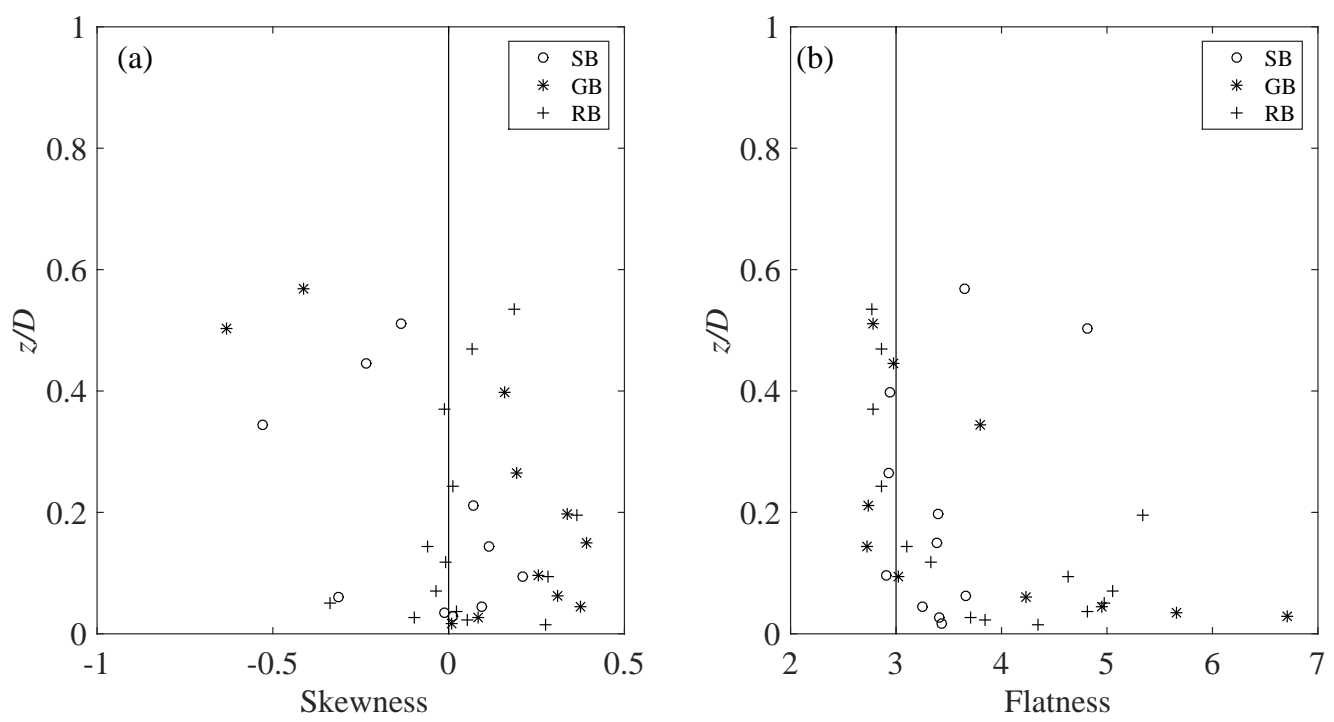

Figure 17 Skewness (a) and flatness (b) of the fluctuating component $v_{x}^{\prime} . V_{D A}=0.045 \mathrm{~m} / \mathrm{s} ; H=0.105 \mathrm{~m} ; T=1.2 \mathrm{~s}$.

Willert (2015) in a wind tunnel in the presence of grid-generated turbulence.

In Fig. 17 the skewness and flatness of fluctuating component $v_{x}^{\prime}$ for a wave plus current case characterized by $V_{D A}=0.045 \mathrm{~m} / \mathrm{s}, H=0.105 \mathrm{~m}$ and $T=1.2 \mathrm{~s}$ are shown for all the roughness conditions. Differently from what happens in the current only case, the skewness is mainly positive in the lower part of the water column for most of the measured points in the case of sandy and gravel bed, while it changes sign in the upper part of the measured column. In the rippled bed case it remains close to zero for most of the points, showing a slightly positive skewness in the upper part of the measured region. The flatness is rather similar for all the three roughness conditions. It takes large values near the bottom, while at high elevations from the bed it assumes values close to 3. However, at some higher elevations the flatness may be much larger than 3 for the sand bed. On the basis of the results shown in Fig. 17, close to the bed the distribution is far from a Gaussian distribution in the gravel and rippled bed cases, while it follows better the normal distribution in the sand bed case. This is also confirmed by the results on the $v_{x}^{\prime}$ distributions shown in Fig. 13, where in the GB and RB cases the peakedness is much higher than the normal one. The normal distribution may be attained far from the bed when the skewness is close to zero.

\subsection{Analysis of phase-averaged quantities}

The analysis of phase-averaged velocities has been performed in order to show how the velocity in the current direction varies during the cycle due to the superposition of an orthogonal wave with the current. In Fig. 18 the phase-averaged current-directed velocity profiles acquired along the water column are plotted at eight different instants within the wave cycle, namely $\phi=\pi / 4$, $\pi / 2,3 \pi / 4, \pi, 5 \pi / 4,3 \pi / 2,7 \pi / 4$ and $2 \pi$. Phase equal to zero corresponds to the zero-upcrossing wave. In each subplot the $\mathrm{SB}, \mathrm{GB}$ and $\mathrm{RB}$ (at the trough) tests with a depth averaged velocity $V_{D A}=0.045 \mathrm{~m} / \mathrm{s}$, a wave height $\mathrm{H}=0.085 \mathrm{~m}$ and a period $\mathrm{T}=1.2 \mathrm{~s}$ are reported. Close to the bed, $\mathrm{SB}, \mathrm{GB}$ and RB data show some differences: indeed when the bottom is rough (GB) the velocity gradient close to the wall is smaller, probably as a consequence of the larger resistance generated at the bottom by the macro-roughness in the presence of the waves. The SB tests, due to the very small bed roughness, independently on the considered phase, exhibit the largest near-bottom velocity gradient; overall close to the bed the RB tests show velocities similar to those of the SB 
tests apart from $\phi=3 \pi / 2$ and $\phi=7 \pi / 4$, where the RB case shows the largest near bed velocity. At higher elevations the RB velocity profiles are similar to those of the SB case; however when the velocities attain the maximum values $(\phi=3 / 4 \pi \div \pi)$, there is a large difference between them. At all the phases the RB profiles show a strong velocity gradient near the bed, that at an elevation comparable to the ripple height, abruptly decrease. The constancy of the velocity profiles in the rippled bed case for $2 \mathrm{~cm}<z<16 \mathrm{~cm}$ can be explained by the vertical transfer of momentum due to the vortices ejected at the ripple crests that tend to homogenize the velocity profiles. This occurrence was already observed by Faraci et al. (2008), who pointed out that far from the bed ripples behave like a macro-roughness, due to the strong turbulence associated to the high vorticity released at the ripple crests.

The GB profiles close to the bed exhibit negative velocities at all the accelerating phases. Such behaviour has been noticed in all the GB tests, thus excluding the possibility that this effect could be due to local disturbances. Further analyses are still needed in order to explain such phenomenon.

In general the velocity profiles exhibit a significant variability in relation to the phase, reaching the minimum value close to a phase equal to $\pi / 3$, and the maximum value at $1.47 \pi$. This result is in agreement with the mechanism responsible for the time variability of the current velocity described in section 3.1 according to which the minimum of $V_{x}$ should fall close to the wave crest and the maximum close to the wave trough.

When the wave superimposes the existing current, the water depth is obviously greater under the wave crest and lower under the trough. Thus, since the volume of water flowing across the section is constant with time, the velocity assumes a larger value when the cross section is smaller, i.e. during the trough passage and vice versa.

The phase-averaged analysis within the wave cycle has also been performed at the different measurement points along the ripple profile in Fig. 19 at the following phases $\phi: \pi / 4, \pi / 2,3 \pi / 4, \pi$, $5 \pi / 4,3 \pi / 2,7 \pi / 4$ and $2 \pi$. Due to the similarity of the results at the two crests, only those acquired at the onshore crest (point 5) have been reported along with the other three points $(2,3$ and 4 , see Fig. 1 for reference). As regards the phases at which the velocity attains the maximum, the same considerations which have been observed for Fig. 18 still apply. Fig. 19 shows that during the positive wave half cycle only slight differences between the different locations can be observed. In the negative wave half cycle, when the current velocity decreases in time the differences increase. More specifically the current velocity at point 2 decreases slightly more rapidly than at the other three points. Near the bed the lowest velocities are always exhibited by points 4 and 5 throughout the wave cycle. This latter evidence may be related to the existence of recirculating cells that are particularly evident at those two positions, as it can be confirmed by looking at Fig. 5 of Faraci et al. (2008).

\section{Conclusions}

In this work the statistics of the current directed velocity component under a wave plus current flow in the presence of a sandy bed, a gravel bed and a rippled bed have been investigated. The analysis has been performed on the basis of experimental data already acquired by the authors but never considered in this perspective.

The current velocity has been split into a time-averaged velocity, an oscillating component and a turbulent fluctuation.

Firstly, the probability density function of the total near bed velocity has been examined. It has been found that in the current only case, the probability distribution function of the near bed velocity is closely similar to the Gaussian one, with slight differences between sand, gravel and rippled bed. When the waves are superimposed onto the current such agreement is lost, and it has been observed that the PDF shows double peaks which separation distance in terms of velocity is a function of the wave kinematics (i.e. relative water depth and wave steepness). The decoupling of 

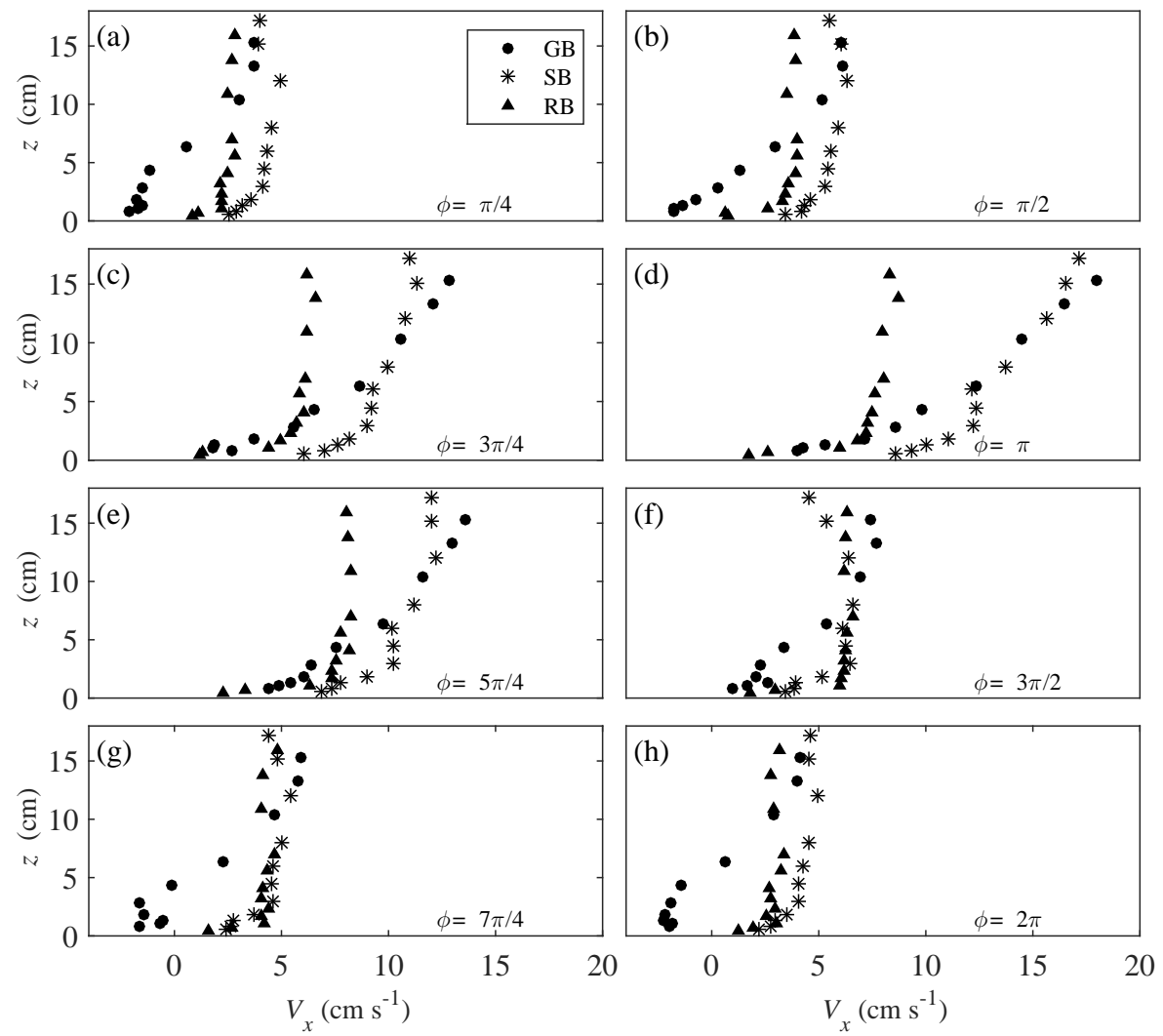

Figure 18 Analysis of phase averaged wave plus current flow (a) $\pi / 4 ;$ (b) $\pi / 2 ;$ (c) $3 \pi / 4 ;$ (d) $\pi$; (e) $5 \pi / 4 ;$ (f) $3 \pi / 2 ;$ (g) $7 \pi / 4$; (h) $2 \pi$. Experimental conditions as in Fig. 8.

the current velocity in two classes characterized by events occurring in concurrence with onshore or offshore wave velocities or upward or downward velocities especially in the rippled bed case is crucial to explain such process. Indeed in this way, two classes of data can be clearly distinguished. The peak of PDF of the current velocity associated with onshore directed wave velocities $V_{x}^{y+}$ always occurs at smaller velocities than that of $V_{x}^{y-}$. This occurrence is explained by the mass conservation principle consequent to the increasing of the cross section under the crest passage and also by a vertical advection of x-momentum. Along the ripple profile, however, the occurrence of stronger or weaker current velocities is affected by the vorticity dynamics associated with the recirculating cells separating at the ripple crest.

The statistical analysis of the turbulent fluctuations in the wave plus current case showed that the double peaks disappear from PDFs, since the oscillating component is not included any more. The PDF is close to a normal distribution in the sand bed case, while in the gravel and rippled bed cases although the distribution is close to be symmetric, it exhibits a flatness significantly larger than 3. Reynolds stresses are mostly negative both in the current only and in wave plus current conditions. In this last case however, the minima almost double and are located at higher elevations. Moreover high order statistics of the turbulent velocity fluctuations generated by super- 

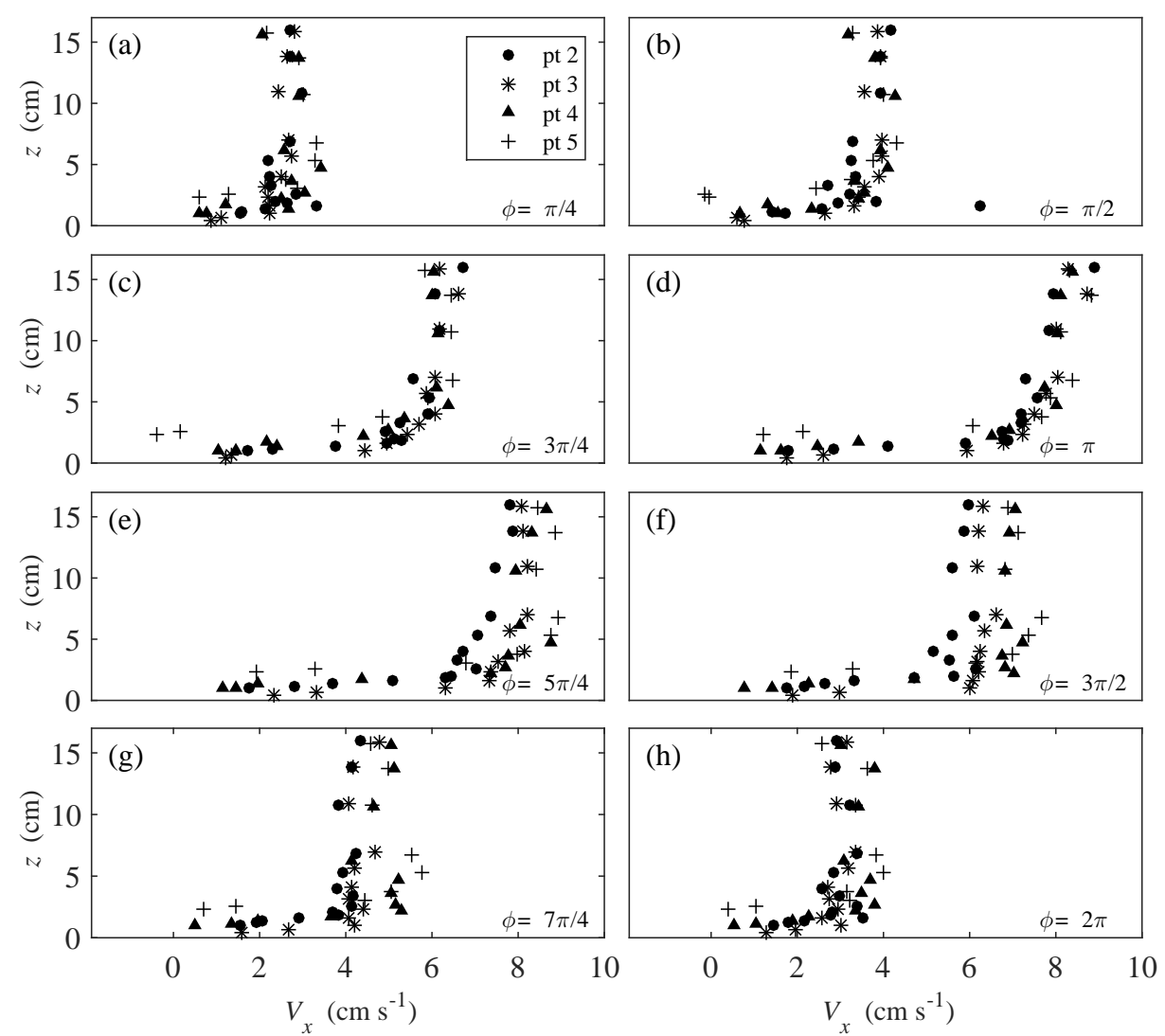

Figure 19 Analysis of phase averaged wave plus current flow for the rippled bed case (a) $\pi / 4$; (b) $\pi / 2$; (c) $3 \pi / 4$; (d) $\pi$; (e) $5 \pi / 4$; (f) $3 \pi / 2$; (g) $7 \pi / 4$; (h) $2 \pi . \mathrm{z}=0$ is set at the ripple trough. Experimental conditions as in Fig. 8.

imposing an orthogonal regular wave on a current have been investigated in order to understand how waves influence the current properties and the turbulence structure in the presence of different bed roughness conditions.

The aforementioned decoupling of the velocity events in terms of the positive and negative wave velocity indicates the influence of the wave phase on the current velocity. For this reason a phase analysis has also been performed. The superposition of the waves on the currents strongly influences the velocity profiles in the current direction, which exhibit a significant variability in relation to the phase. Indeed they reach higher values in the second half cycle, corresponding to the passage of the wave trough, as a consequence of mass conservation principle coupled with a convection of $\mathrm{x}$-momentum in the vertical direction; the latter causes the anticipation of the phase at which the maximum of the current velocity occurs.

The results of the present work suggest that the use of a more detailed statistical characterization along with a thorough description of the phase variability of near bed velocity could certainly contribute to a better modelling of coastal sediment transport and related processes. 


\section{Acknowledgements}

This work has been financed by Italian Ministry of University, Education and Research, PRIN 2012 Project "Hydro-morphodynamics modelling of coastal processes for engineering purposes". EC funded project Hydralab PLUS is also acknowledged.

\section{References}

Andersen, K. H., \& Faraci, C. (2003). The wave plus current flow over vortex ripples at an arbitrary angle. Coastal Engineering, 47, 431-441. (doi: 10.1016/S0378-3839(02)00158-8)

Arnskov, M. M., Fredsøe, J., \& Sumer, B. M. (1993). Bed shear stress measurements over a smooth bed in three-dimensional wave-current motion. Coastal Engineering, 20, 277-316.

Cheng, N. S. (2006). Influence of shear stress fluctuation on bed particle instability. Physics of Fluids, 18(9).

Faraci, C., Foti, E., Marini, A., \& Scandura, P. (2012). Waves plus currents crossing at a right angle: Sandpit case. Journal of Waterway, Port, Coastal, and Ocean Engineering, 138(5), 339-361. (doi: 10.1061/(ASCE)WW.1943-5460.0000140)

Faraci, C., Foti, E., \& Musumeci, R. (2008). Waves plus currents crossing at a right angle: the rippled bed case. Journal of Geophysical Research, 113(C07018), 1-26. (doi: 10.1029/2007JC004468)

Fernando, P. C., Guo, J., \& Lin, P. (2011). Wave - current interaction at an angle 1: experiments. Journal of Hydraulic Research, $49(4), 424-436$.

Fredsøe, J., Andersen, K. H., \& Sumer, B. M. (1999). Wave plus current over a ripple-covered bed. Coastal Engineering, 38, 177-221.

Friedrich, H., Nikora, V., Melville, B. W., \& Coleman, S. E. (2006). Statistical interpretation of geometric differences in ripple and dune shapes. In 7th int. conf. on hydroscience and engineering. IAHR.

Grant, W. D., \& Madsen, O. S. (1979). Combined wave and current interaction with a rough bottom. Journal of Geophysical Research., 84(C4), 1797-1808.

Grass, A. J., \& Ayoub, R. N. M. (1983). Bed load transport of fine sand by laminar and turbulent flow. In 18th int. conf. on coastal engineering (pp. 1589-1599). ASCE.

Huang, Z., \& Mei, C. C. (2003). Effects of surface waves on a turbulent current over a smooth or rough seabed. Journal of Fluid Mechanics., 497, 253-287.

Kim, J., Moin, P., \& Moser, R. (1987). Turbulence statistics in fully developed channel flow at low reynolds number. Journal of Fluid Mechanics, 177, 133-166.

Lim, K. Y., \& Madsen, O. S. (2016). An experimental study on near-orthogonal wave-current interaction over smooth and uniform fixed roughness beds. Coastal Engineering, 116, 258274.

Lim, K. Y., Madsen, O. S., \& Cheong, H. F. (2012). Current characteristics in the presence of near orthogonal waves. In 33rd int. conf. on coastal engineering. ASCE.

Lodahl, C. R., Fredsøe, J., \& Sumer, B. M. (1998). Turbulent combined oscillatory flow and current in a pipe. Journal of Fluid Mechanics, 373, 313-348.

Madsen, O. S., Negara, A. S., Lim, K. Y., \& Cheong, H. F. (2010). Near-bottom flow characteristicsof currents at arbitrary angle to $2 \mathrm{~d}$ ripples. In 32nd int. conf. on coastal engineering.

Mathiesen, P. P., \& Madsen, O. S. (1996). Waves and current over a fixed ripples bed: 1. bottom roughness experienced by currents in the presence of waves. Journal of Geophysical Research, $101(\mathrm{C} 7), 16543-16550$.

Musumeci, R. E., Cavallaro, L., Foti, E., Scandura, P., \& Blondeaux, P. (2006). Waves plus currents crossing at a right angle. Experimental investigation. Journal of Geophysical Research, 111 (C07). (doi: 10.1029/2005JC002933) 
Nikora, V. I., Goring, D. G., \& Biggs, B. J. F. (1998). On gravel bed roughness characterization. Water Resources Research, $34(3), 517-527$.

Parker, G., Paola, C., \& Leclair, S. (2000). Probabilistic exner sediment continuity equation for mixtures with no active layer. Journal of Hydraulic Engineering, 126(818).

Ranasoma, K. I. M., \& Sleath, J. F. A. (1994). Combined oscillatory and steady flow over ripples. Journal of Waterway, Port, Coastal and Ocean Engineering, 120(4), 331-346.

Scandura, P., Faraci, C., \& Foti, E. (2016). A numerical investigation of acceleration-skewed oscillatory flows. Journal of Fluid Mechanics, 808, 576613.

Simons, R. R., Grass, T. J., \& Mansour-Tehrani, M. (1992). Bottom shear stresses in the boundary layer under waves and currents crossing at right angle. In 23rd int. conf. on coastal engineering (Vol. 1, pp. 604-617). ASCE.

Simons, R. R., Grass, T. J., Saleh, W. M., \& Mansour-Tehrani, M. (1994). Bottom shear stresses under random waves with a current superimposed. In 24th int. conf. on coastal engineering. ASCE.

Willert, C. E. (2015). High-speed particle image velocimetry for the efficient measurement of turbulence statistics. Experiments in Fluids, 56(17).

Yuan, J., \& Madsen, O. (2015). Experimental and theoretical study of wave-current turbulent boundary layers. Journal of Fluid Mechanics, 765, 480523. 